\title{
In der Schwebe
}

Markt, Staat und Wettbewerb in Deutschland zwischen I 918 und 1948

von Franz Hederer und Kim Christian Priemel

\section{Einführung}

Wer die Frage nach den Konturen der Wirtschaftsordnung in der ersten Hälfte des 20.Jahrhunderts stellt, muss nicht befürchten, ohne Antwort zu bleiben. Im Gegenteil sieht man sich mit einer Fülle an Deutungsangeboten konfrontiert, welche den Wandel im Verhältnis privater und staatlicher Interessen ausleuchten. Die hundertjährige Wiederkehr von Erstem Weltkrieg, Revolution und Weimarer Verfassung scheint die Auseinandersetzung mit der Zwischenkriegszeit ebenso stimuliert zu haben, wie die vielfältigen Krisendiagnosen der vergangenen Jahre. ${ }^{\mathrm{I}}$

Ein systematischer Überblick indes liefert drei auffällige Befunde: Zum einen dominiert eine konventionelle, an den Brüchen der ersten Hälfte des 20.Jahrhunderts orientierte Periodisierung, in deren Kontext die jeweilige Wirtschaftsordnung als Facette eines umfassenderen politischen Prozesses erscheint. ${ }^{2}$ Zum anderen ist die Wahrnehmung nach wie vor geprägt von den bereits zeitgenössisch virulenten Narrativen einer „organisierten“, „geplanten“ und staatlich geregelten Wirtschaftsordnung ${ }^{3}$, die ihrerseits als Symptom einer epochalen Transformationsdynamik „des

I So gab die Finanzkrise seit 2008 Anlass zu Vergleichen mit der Great Depression der späten I $920 e r$ Jahre, etwa Jan-Otmar Hesse/Roman Köster/Werner Plumpe, Die Große Depression. Die Weltwirtschaftskrise I929-I939. Bonn 2015, 205-2 I6, oder Albrecht Ritschl, War 2008 das neue I929? Richtige und falsche Vergleiche zwischen der Großen Depression der I 930er Jahre und der Großen Rezession von 2008, in: Perspektiven der Wirtschaftspolitik I3, 2012, 36-57.

2 Andere Zäsuren (I923/24, I936, I948) setzen hingegen wirtschaftshistorische Zugänge, darunter die klassische Studie von Dietmar Petzina, Autarkiepolitik im Dritten Reich. Der nationalsozialistische Vierjahresplan. Stuttgart I 968; Gerald D. Feldman, The Great Disorder. Politics, Economics, and Society in the German Inflation, I9I4-I924. New York I993, und Harold James, Deutschland in der Weltwirtschaftskrise I924-I936. Stuttgart I988. Vgl. auch Albrecht Ritschl, Deutschlands Krise und Konjunktur I924-I934. Binnenkonjunktur, Auslandsverschuldung und Reparationsproblem zwischen Dawes-Plan und Transfersperre. Berlin 2002, und Tim Schanetzky, „Kanonen statt Butter“. Wirtschaft und Konsum im Dritten Reich. München 20I5, I47-I60.

3 Etwa Hans-Jürgen Puhle, Historische Konzepte des entwickelten Industriekapitalismus. „Organisierter 
Kapitalismus“ schlechthin gelesen wurde. ${ }^{4}$ Die Aussagekraft dieser Großkonzepte ist heute, nicht zuletzt angesichts einer Vielzahl empirischer Detailstudien zu Politik, Ökonomie und ihren Akteuren ${ }^{5}$, erheblich erodiert, mehr von assoziativer denn analytischer Relevanz. ${ }^{6}$ Zum dritten kehren auch revisionistische und kontrovers diskutierte Deutungsversuche regelmäßig zu den Wurzeln einer liberalen political economy zurück, deren Schattierungen variieren, gleichwohl recht stabil zwischen jenen ordo- und sozialliberalen Polen oszillieren, mit denen eine aseptische bundesrepublikanische Vorgeschichte erzählt werden kann. ${ }^{7}$

Was allerdings fehlt, ist der Blick auf mittelfristig persistente Strukturen, die jenseits der radikalen politischen Brüche der deutschen Geschichte den Diskurs über das Verhältnis von Ökonomie und Politik, Privatwirtschaft und Staat, Konkurrenz und Konzentration prägten. Hier setzt der vorliegende Beitrag an und versucht, entlang der Diskussionen um Markt, Wettbewerb und Kartelle die Konturen einer „un-

Kapitalismus“ und „Korporatismus“, in: GG I0, I984, I65-I 84; Dirk van Laak, Planung. Geschichte und Gegenwart des Vorgriffs auf die Zukunft, in: GG 34, 2008, 305-326 und Anselm Doering-Manteuffel, Ordnung jenseits der politischen Systeme: Planung im 20.Jahrhundert, in: GG 34, 2008, 398-406.

mus, in: Franz Boese (Hrsg.), Verhandlungen des Vereins für Socialpolitik in Zürich, I3.-I5. September I928. München I929, 23-4I.

5 Klassisch die Debatte um „Zwangslagen“ der Regierung Brüning und strukturelle Belastungen der Weimarer Wirtschaft: Zusammenfassend Albrecht Ritschl, Knut Borchardts Interpretation der Weimarer Wirtschaft. Zur Geschichte und Wirkung einer wirtschaftsgeschichtlichen Kontroverse, in: Jürgen Elvert/Susanne Krauß (Hrsg.), Historische Debatten und Kontroversen im I9. und 20.Jahrhundert. Stuttgart 2003, 234-244.

6 Eine Ausnahme mag die neu aufgelegte Grundsatzdebatte um Charakter und Dynamik des „Kapitalismus“ in seiner Doppelbedeutung als historisches Phänomen und analytische Kategorie sein: Vgl. Joyce Appleby, The Relentless Revolution. A History of Capitalism. New York 20 ıо; Jürgen Kocka, Geschichte des Kapitalismus. Bonn 2013; Werner Plumpe, Das kalte Herz. Kapitalismus: Die Geschichte einer andauernden Revolution. Berlin 20I9; Jens Beckert, Imaginierte Zukunft. Fiktionale Erwartungen und die Dynamik des Kapitalismus. Frankfurt a. M. 2018.

7 Etwa bei Jens Hacke, Existenzkrise der Demokratie. Zur politischen Theorie des Liberalismus in der Zwischenkriegszeit. Frankfurt a. M. 20г 8, und, deutlich kontroverser, bei Tim B. Müller, Die Geburt des SozialLiberalismus aus dem Geist der Verwaltung. Zur Erfindung der modernen Wirtschaftspolitik in der Weimarer Republik, in: Anselm Doering-Manteuffel/Jörn Leonhard (Hrsg.), Liberalismus im 20. Jahrhundert. Stuttgart 20I5, I27-I55; ders., Demokratie und Wirtschaftspolitik in der Weimarer Republik, in: VfZ 62, 20I4, 569-60I. Vgl. stellvertretend die wirtschaftshistorische respektive demokratietheoretische Kritik von Roman Köster, Keine Zwangslagen? Anmerkungen zu einer neuen Debatte über die deutsche Wirtschaftspolitik in der Großen Depression, in: VfZ 63, 20I5, 24I-257, und Steffen Kailitz, Demokratie und Wirtschaftspolitik in der Weimarer Republik in international vergleichender Perspektive, in: VfZ 63, 20I5, 437-45I. 
ausgetragenen“8 Wirtschaftsordnung freizulegen, in der grundlegende Fragen über politische, soziale oder moralische Wert- und Ordnungsvorstellungen verhandelt wurden. ${ }^{9}$ Die von den Zeitgenossen auf unterschiedlichen Ebenen intensiv geführte Debatte wird im Folgenden als Suche nach einem kohärenten Strukturprinzip der Wirtschaft interpretiert, die erst nach I945 zu einem (vorläufigen) Abschluss gebracht wurde. ${ }^{\text {Io }}$

Ziel des Beitrages ist es, zentrale, zugleich heterogene und wechselseitig überlappende Entwicklungslinien in den Vorstellungen von wirtschaftlicher Ordnung sichtbar zu machen: Dazu wird zunächst die ökonomisch fragile Situation nach Ende des Ersten Weltkriegs als zweifache, gleichermaßen praktische wie theoretische Herausforderung interpretiert, vor deren Hintergrund auch die „hybride“ “II Rolle des in der Forschung zumeist als Scheinriese qualifizierten Reichswirtschaftsrates (RWR) neu vermessen wird. ${ }^{\text {I2 }}$ Ein dritter Abschnitt widmet sich der Diskussion um Kartelle und Wettbewerb in der Weimarer Republik aus vornehmlich juristischer Perspektive, die ein gewandeltes sozialmoralisches Verständnis von Wirtschaftsmacht dokumentiert. ${ }^{\text {I3 }}$ Viertens wurde um I930 die Frage nach dem Verhältnis von privater und staatlicher Macht in der Wirtschaft ganz konkret auf die Tagesordnung gesetzt, wovon besonders plastisch die Debatte um „Deutschland als Wirtschafts-

8 Der Begriff in Anlehnung an Helmut Neuhaus, Reichsständische Repräsentationsformen im I6.Jahrhundert. Reichstag - Reichskreistag - Reichsdeputationstag. Berlin I982, 522.

9 Vgl. Roman Köster, Die Wissenschaft der Außenseiter. Die Krise der Nationalökonomie in der Weimarer Republik. Göttingen 20I I, hier 269-306.

Io Dazu auch Kim Christian Priemel, Searching for Order. German Jurists Debate Economic Power, c. I9I9-I949, in: Moritz Föllmer/Pamela Swett (Eds.), Cultures of Capitalism. Cambridge 2020 (in Vorbereitung).

I I Alexander Nützenadel, Die Stunde der Ökonomen. Wissenschaft, Politik und Expertenkultur in der Bundesrepublik I949-I974. Göttingen 2005, hier I39.

I2 Das Bild des RWR in der Forschung ist so einhellig wie ernüchternd. Stellvertretend Gerhard A. Ritter, Die Entstehung des Räteartikels I 65 der Weimarer Reichsverfassung, in: HZ 258, I 994, 73-I I 2, hier 74 („,totgeboren") oder Ulrich Nocken, Korporatistische Theorien und Strukturen in der deutschen Geschichte des I9. und 20.Jahrhunderts, in: Ulrich von Alemann (Hrsg.), Neokorporatismus. Frankfurt a. M. I98I, I7-39, hier 36 („Schattendasein“). Jüngst Alfred Reckendrees, Weimar Germany: The First Open Access Order that Failed? In: Constitutional Political Economy 26, 20I 5, 38-60, hier 49. Einen fundierten Überblick bietet Joachim Lilla, Der Vorläufige Reichswirtschaftsrat I920-I 933/34. Zusammensetzung - Dokumentation - Biographien. Düsseldorf 20I2. Knapp Franz Hederer, Art.Reichswirtschaftsrat, in: Handwörterbuch zur deutschen Rechtsgeschichte. Bd. IV. 2.Aufl. 2020 (im Erscheinen).

I3 Vgl. die in der Rechtsgeschichte einflussreichen, hinsichtlich ihrer normativen Setzungen aber problematischen Arbeiten von Knut Wolfgang Nörr, Die Leiden des Privatrechts. Kartelle in Deutschland von 
staat“I4 zeugte, die im Folgenden entlang der rechtswissenschaftlichen, erst di-, dann wieder konvergierenden Arbeiten Heinrich Kronsteins und Franz Böhms sowie ihrer Zeitgenossen Hans Carl Nipperdey und Walter Eucken nachgezeichnet wird. ${ }^{15}$ Hiervon ausgehend eröffnen sich fünftens Kontinuitäten bis in die frühe Bundesrepublik, die die Bedeutung personeller Netzwerke und kontingenter Konstellationen gegenüber der intrinsischen Kraft ,besserer‘ Ideen akzentuieren. Sichtbar wird so eine überraschend kohärente, gleichwohl hart geführte Debatte über ökonomische Macht und Wettbewerb in der ersten Hälfte des 20.Jahrhunderts.

\section{Ordnungsverlust: Der Erste Weltkrieg und die Folgen}

Das Ende des Ersten Weltkriegs warf das Problem wirtschaftlicher Ordnung in fundamentaler Weise auf: Zum einen war unklar, in welche zukünftige Volks- die Kriegswirtschaft transformiert werden sollte. Zum anderen galt es, handfeste ökonomische Schwierigkeiten ungekannten Ausmaßes zu meistern, die von der Umstellung auf die Bedürfnisse einer Friedenswirtschaft über Gebietsverlust und Reparationsleistungen bis zur galoppierenden Geldentwertung reichten. ${ }^{16}$ Zwischen Theorie und Praxis bestand freilich ein ebenso erwartbares wie erhebliches Spannungsverhältnis. Die populären Forderungen nach umfangreichen Sozialisierungen $^{17}$ oder einer Ergänzung bzw. gar Ersetzung des parlamentarischen Systems

der Holzstoffkartellentscheidung zum Gesetz gegen Wettbewerbsbeschränkungen. Tübingen I994, und ders., Zwischen den Mühlsteinen. Eine Privatrechtsgeschichte der Weimarer Republik. Tübingen I 988.

I4 Vor allem: Ernst RudolfHuber, Das Deutsche Reich als Wirtschaftsstaat. Tübingen I 93 I; Walter Eucken, Staatliche Strukturwandlungen und die Krisis des Kapitalismus, in: Weltwirtschaftliches Archiv 36, I932, 297-32 I; Carl Schmitt, Das Zeitalter der Neutralisierungen und Entpolitisierungen, in: Ders., Der Begriff des Politischen. Text von I 932 mit einem Vorwort und drei Corollarien. 7.Aufl. Berlin 2002, 79-95. Hierzu jetzt auch Hacke, Existenzkrise (wie Anm. 7), 335-366.

I5 Franz Böhm, Das Problem der privaten Macht. (Ein Beitrag zur Monopolfrage), in: Die Justiz 3, I $927 / 28$, 324-345; ders., Wettbewerb und Monopolkampf. Eine Untersuchung zur Frage des wirtschaftlichen Kampfrechts und zur Frage der rechtlichen Struktur der geltenden Wirtschaftsordnung. Baden-Baden 20 Io (Erstaufl. Berlin I933).

I6 Im Überblick: Feldman, Great Disorder (wie Anm. 2); Dieter Ziegler, Die Kriegswirtschaft im Ersten Weltkrieg - Trends der Forschung, in: JbWG 56, 20I 5, 313-323; Carl Ludwig Holtfrerich (Hrsg.), Das Reichswirtschaftsministerium der Weimarer Republik und seine Vorläufer. Strukturen, Akteure, Handlungsfelder. Berlin 2016.

I7 Vgl. etwa die Verhandlungen des Vereins für Sozialpolitik in Regensburg I9I9. München I920. Klaus 
durch Rätegremien oder berufsständische Organe sind hinlänglich bekannt, werden zumeist aber eher überschätzt. Denn viele der Nachkriegskonzepte einer Reorganisation von ,Wirtschaft" und ,Staat" verband vor allem ihr inhaltlich amorpher, ideologisch umkämpfter und tagespolitisch eher nachrangiger Charakter. Zwar teilten sie eine durchaus kohärente Zeitdiagnose, der zufolge die Wirtschaft nicht länger dem Treiben Einzelner allein überlassen werden durfte, von einer fundamentalen Revision des privatrechtlichen Prinzips konnte indes kaum die Rede sein. ${ }^{18}$ Im Kern sanktionierte die Weimarer Reichsverfassung (WRV) ungeachtet aller sozialistischen, gemeinwirtschaftlichen oder ständischen Rhetorik die liberale Privatrechtsordnung, wenn auch unter einfachgesetzlichem Vorbehalt. ${ }^{\text {I9 }}$

Entscheidend war in der Praxis damit vielmehr, welche konkreten Politikvorhaben im Reichstag Mehrheiten finden und auf Implementierung in der Praxis hoffen konnten; angesichts der enormen Probleme und unterschiedlichen Interessenlagen ein hart umkämpftes Feld. Die intendierte, aber nur partiell realisierte Verlagerung wirtschaftspolitischer Entscheidungsfindung in den RWR als „unpolitisch“ konzipiertem Organ einerseits, der (keineswegs erst seit den I930er Jahren praktizierte) Rekurs auf das Instrument der Notverordnungen andererseits boten so zwei unterschiedliche Ausweichstrategien, um die ökonomischen Herausforderungen praktisch angehen zu können. ${ }^{20}$ Dass sich die autoritäre Notverordnungspraxis gegenüber einem pluralistisch konzipierten,Wirtschaftsparlament‘ als Mittel der Wahl

\footnotetext{
Novy, Strategien der Sozialisierung. Die Diskussion der Wirtschaftsreform in der Weimarer Republik. Frankfurt/New York 1978. Vgl. auch zur „Gemeinwirtschaft“ RudolfWissell/Wichardvon Moellendorff, Wirtschaftliche Selbstverwaltung. Zwei Kundgebungen des Reichswirtschaftsministeriums. Jena I9I9. Vgl. Akten der Reichskanzlei (AdR), Kabinett Bauer, Bd. I, Nr.20, Kabinettssitzung vom 8.Juli i9i 9, 92. Vgl. auch Friedrich Glum, Rez. Huber, Wirtschaftsstaat [u.a.], in: Archiv des öffentlichen Rechts 64, I934, I Io-I 23, hier III.

I8 Plakativ, aber nicht repräsentativ Walter Rathenau, Die neue Wirtschaft. Berlin I9I 8, 5. Ähnlich Rudolf Hilferding, Die Aufgaben der Sozialdemokratie in der Republik. Berlin [I 927], 5. Auch Carl-Ludwig Holtfrerich, Aus dem Alltag des Reichswirtschaftsministeriums während der großen Inflation I9I9-I923/24, in: Ders. (Hrsg.), Reichswirtschaftsministerium (wie Anm. 16), 224-360, besonders 254f. Vgl. Plumpe, Herz (wie Anm.6), 31 2-327.

I9 So dezidiert Huber, Wirtschaftsstaat (wie Anm. I4); anders Franz Neumann, Die soziale Bedeutung der Grundrechte in der Weimarer Verfassung, in: Die Arbeit 7, I930, H. 9, 569-582. Vgl. auch Franz Hederer, Wirtschaftsordnung, in: Rüdiger Voigt (Hrsg.), Aufbruch zur Demokratie. Die Weimarer Verfassung als Bauplan für eine demokratische Republik. Baden-Baden 2020 (in Vorbereitung).

20 Vgl. bereits Hugo Sinzheimer, Ein Arbeitstarifgesetz. Die Idee der sozialen Selbstbestimmung im Recht [I9I6]. 2.Aufl. Tübingen I977, besonders I97-202.
} 
erweisen sollte, verrät allerdings viel über das zeitgenössische Bedürfnis nach Eindeutigkeit.

Die theoretische Suche nach Ordnung wurde somit meist von der Praxis überlagert - und nicht selten überholt. Denn aus Sicht der politischen Akteure ging es schon bald nicht mehr darum, die ökonomischen Verhältnisse grundlegend umzugestalten, sondern die tagesaktuellen Herausforderungen zwischen Inflation und Reparation einigermaßen zu meistern und das auf die Kriegswirtschaft zurückgehende Knäuel von Wirtschaft, Politik und Staat zu entwirren. Während jedoch unmittelbar nach Kriegsende der Zugriff des Staates auf die Privatwirtschaft im Vordergrund stand, sollte sich die Perspektive bis I930 ganz erheblich verschieben: Nun waren es die Privaten selbst, die im Bestreben, den Unbilden des wirtschaftlichen Wettbewerbs zu entfliehen, wie öffentliche Einrichtungen und mitunter gar wie Hoheitsträger agierten. ${ }^{2 \mathrm{I}}$ Die in der Verfassung garantierte Gewerbefreiheit konkurrierte folglich mit der ebenfalls verfassungsrechtlich verankerten Vertragsfreiheit. Der Konzentrations- und Kartellierungsprozess der deutschen Wirtschaft gründete damit im liberalen Fundament der Weimarer Wirtschaftsordnung und untergrub es zugleich.

Dieser Gegensatz, der den vor I9I4 begonnenen und sich im Krieg zuspitzenden Trend zur (Zwangs-)Organisation reflektierte, zog nicht nur unter Nationalökonomen und Politikern, sondern auch unter Juristen Aufmerksamkeit auf sich. Während Arthur Nussbaum, einer der ersten Rechtswissenschaftler, der sich mit einem Syntheseversuch vorwagte, I920 befand, es habe „für die ganze Kulturwelt ein neuer Abschnitt der Rechtsentwicklung“ begonnen ${ }^{22}$, verwiesen andere Beobachter auf weit längere Entwicklungen. ${ }^{23}$ So beschrieb der angesehene Wirtschaftsjurist Karl Geiler in einer Reihe von Vorträgen, die er zwischen I9I9 und I 922 hielt, einen tiefgreifenden „Umwandlungsprozess“, der traditionelle liberale Auffassungen von

\footnotetext{
2 I Vgl. den Abschlussbericht zu den Erzeugungs- und Absatzbedingungen der deutschen Wirtschaft. Auf Grund der Veröffentlichungen des Enquete-Ausschusses zusammengestellt durch B. Dernburg et al. Berlin I93I.

22 Arthur Nussbaum, Das neue deutsche Wirtschaftsrecht. Eine systematische Übersicht über die Entwicklung des Privatrechts und der benachbarten Rechtsgebiete seit Ausbruch des Weltkrieges. Berlin I920, I.

23 Zu Entwicklungen vor I9I4 u.a. Rudolf Piepenbrock, Der Gedanke eines Wirtschaftsrechts in der neuzeitlichen Literatur bis zum Ersten Weltkrieg. Köln 1964, und Mathias Schmoeckel/Matthias Maetschke, Rechtsgeschichte der Wirtschaft seit dem I9. Jahrhundert. 2. Aufl. Tübingen 2016.
} 
Staat und Wirtschaft strukturell in Frage stellte. Und obschon dem bürgerlichen Wirtschaftsanwalt sozialrevolutionäre Attitüden fernlagen, erkannte er als drängendes Problem vor allem den Zuwachs privater wirtschaftlicher Macht und die darin enthaltenen „Gefahr für die Allgemeinheit“. ${ }^{24}$

Geiler und Nussbaum standen mit ihren Diagnosen - nicht notwendigerweise auch mit ihren Rezepten - keineswegs allein auf weiter Flur, sondern festen Fußes im Mainstream. Das wirtschaftsrechtliche Feld boomte in Weimar geradezu, und seine Institutionalisierung wie auch Ausdifferenzierung reflektierten das wachsende juristische und ökonomische Bewusstsein dafür, den vielfältigen, intrikaten Verbindungen von Staat und Wirtschaft konzeptionell beikommen zu müssen. ${ }^{25}$ Bemerkenswert war vor allem die schul- und ideologieübergreifende Einigkeit, die in diesem Punkt bestand: Liberale und konservative, sozialdemokratische und antidemokratische Stimmen von Hugo Sinzheimer über Gustav Radbruch bis Carl Schmitt stimmten darin überein, dass die Privatisierung des Staatlichen und die Verstaatlichung des Privaten, insbesondere des ökonomischen Lebens, ein epochales Signum darstellten. ${ }^{26}$ Begriffe wie „Wirtschaftsmacht“, „Wirtschaftsstaat“, „Verwirtschaftlichung“ sowie ihre Derivative befanden sich seit den I89oer Jahren im Aufwind und verbreiteten sich in der Zwischenkriegszeit exponentiell. ${ }^{27}$ Dies war nicht gleichbedeutend damit, dass alle den „Wirtschaftsstaat“ verfassungsrechtlich und praktisch bereits als gegeben sahen, wohl aber dass die Wegschilder in eine nahe Zukunft wiesen, in der „Staatlichkeit im Rahmen ökonomischer Prozesse“ konstru-

24 Karl Geiler, Der genossenschaftliche Gedanke und seine stärkere Verwirklichung im heutigen Wirtschaftsrecht [I92 I], in: Ders., Gesellschaftliche Organisationsformen des neuen Wirtschaftsrechts. 2.Aufl. Mannheim I922, 9I-I Io, hier I09.

25 Schmoeckel/Maetschke, Rechtsgeschichte (wie Anm.23), 225-235, 293-298, 377-38I, 408-4I 2; Nörr, Zwischen den Mühlsteinen (wie Anm.I3); Clemens Zacher, Die Entstehung des Wirtschaftsrechts in Deutschland. Berlin 2002, 24-47, 96-IOI.

26 Vgl. Florian Meinel, Der Jurist in der industriellen Gesellschaft. Ernst Forsthoff und seine Zeit. Berlin 20I2, I02. Zu Sinzheimer vgl. Michel Coutu, Autonomie collective et pluralisme juridique: Georges Gurvitch, Hugo Sinzheimer et le droit du travail, in: Droit et Societé 90, 2015, 35I-372, und Jens Meierhenrich, The Remnants of the Rechtsstaat. An Ethnography of Nazi Law. Oxford 2018, 6off. Zu Radbruch: Marc André Wiegand, Ökonomie, Ideologie, Rechtsphilosophie. Zum Verhältnis von Wirtschaft und Recht bei Gustav Radbruch, in: Martin Borowski/Stanley L. Paulson (Hrsg.), Die Natur des Rechts bei Gustav Radbruch. Tübingen 20I5, 53-74, hier 62-73. Zu Schmitt s.u.

27 Vgl. die Ergebnisse einer Suche mit den Eingaben „Wirtschaftsmacht“, „Wirtschaftsstaat“, „wirtschaftliche Macht“, „ökonomische Macht“ und „Marktmacht“, I89o-2000, bei https://books.google.com/ ngrams/ [28.01.2020]. 
iert wurde. ${ }^{28}$ Einen Schritt weiter ging die einflussreiche Fehlerdiagnose Walter Euckens im „Weltwirtschaftlichen Archiv“ von I932. Der bis heute als Gründungsdokument der ordoliberalen Schule geltende Aufsatz ${ }^{29}$, wiewohl mehr Alarmsignal als wissenschaftliches Argument, sah im Einklang mit Schmitt den Weg in den Wirtschaftsstaat infolge staatlicher Kompetenzakkumulation und deren Kapern durch private „Interessenten“ vorgezeichnet. ${ }^{30}$

Hellsichtiger klang ein kurz zuvor an selber Stelle publizierter Aufsatz von Christian Eckert, Kölner Staatswissenschaftler und anders als Eucken wenig später vom NS-Regime in den Ruhestand versetzt. Auch Eckert beschrieb, wie „immer neue Verfilzungen zwischen Staat und Wirtschaft" den Wettbewerb durch private Monopole und autokratische staatliche Maßnahmen ersetzten. Vor allem aber konstatierte er, das zwischen Krieg und Verordnungsregime entstandene deutsche „Notsystem“ folge einem Trend, der für individuelle Freiheit weder Verwendung noch Respekt habe. Seine Äquivalente andernorts, so Eckert, seien Faschismus und Bolschewismus. ${ }^{3 \mathrm{I}}$

\section{Ordnung durch Versachlichung? Der Reichswirtschaftsrat und Weimars political economy}

Der Glaube an eine notwendige Neuordnung des Verhältnisses von Wirtschaft und Staat fand mit dem RWR ihren verfassungsrechtlichen Niederschlag. ${ }^{32}$ Stand die provisorische Reichsregierung der Bildung von Räten gleich welcher Art zu-

28 Huber, Wirtschaftsstaat (wie Anm. I4); vgl. die kritische Rezension von Erich Hula in: Zeitschrift für Nationalökonomie 4, I933, 709; Zitat: Stefanie Middendorf, Staatsfinanzen und Regierungstaktiken: Das Reichsministerium der Finanzen (I9I9-I945) in der Geschichte von Staatlichkeit im 20.Jahrhundert, in: GG 4I, 20I5, I40-I68, hier I47.

29 Köster, Wissenschaft (wie Anm. 9), 30I; Hacke, Existenzkrise (wie Anm. 7), 340; Viktor Vanberg, Liberalismus und Demokratie: Zu einer vernachlässigten Seite der liberalen Denktradition, in: ORDO 65, 20I4, 345-374, hier 35I.

30 Eucken, Strukturwandlungen (wie Anm. I4), 297-32 I, Zitate 299, 302 f., 3I 5, 3I 2.

3I Christian Eckert, Staat und Wirtschaft, in: Weltwirtschaftliches Archiv 35, I932, 357-385, hier 378 und 384 .

32 Friedrich Glum, Der Reichswirtschaftsrat, in: Gerhard Anschütz/Richard Thoma (Hrsg.), Handbuch des Deutschen Staatsrechts, Erster Bd. Tübingen I930, 578-585; Wilhelm Haubold, Die Stellung des Reichswirtschaftsrates in der Organisation des Reiches. Berlin I932; Edgar Tatarin-Tarnheyden, Berufsverbände und Wirtschaftsdemokratie. Ein Kommentar zu Artikel I65 der Reichsverfassung. Berlin I930. 
nächst distanziert gegenüber, so „verankerte“ sie diese nach kontroversen Diskussionen letztlich doch in Art. 65 der Reichsverfassung. ${ }^{33}$ Der Artikel griff Hugo Sinzheimers dualistische Konzeption auf, die den grundsätzlichen Gegensatz zwischen Kapital und Arbeit akzeptierte, gleichzeitig aber das gemeinsame Interesse der Akteure an ökonomischer Prosperität und sozialer Wohlfahrt hervorhob. ${ }^{34}$ Letzteres sollte der künftige RWR verkörpern, der aber von Beginn mit diffusen Erwartungen überfrachtet wurde, die sich aus der weit verbreiteten Skepsis gegenüber der Leistungsfähigkeit des parlamentarischen Systems speisten, und auf eine „Entlastung“ des Reichstags und die „Versachlichung“ der Wirtschaftspolitik zielten. ${ }^{35}$

Der dann I920 aus der Not heraus auf dem Verordnungsweg etablierte, explizit nur „vorläufige“ RWR ${ }^{36}$ bestand aus einem Plenum von 326 Repräsentanten aller als volkswirtschaftlich relevant erachteten Branchen, die sich in zehn Gruppen organisierten, und analog zum Reichstag über ein freies Mandat verfügten. ${ }^{37}$ Anders als in der Verfassung vorgesehen gewährte die Verordnung dem RWR aber kein (voll ausgebildetes) Gesetzesinitiativrecht und verzichtete auch darauf, den Unterbau zu realisieren. Zur Umsetzung von Art. 65 wurde ein Verfassungsausschuss eingerichtet, der I 927 für den RWR einen als konsensfähig erachteten Gesetzentwurf präsentieren, bis 1930 im Reichstag aber nicht die notwendige verfassungsändernde Mehrheit gewinnen konnte. ${ }^{38}$ Der RWR blieb damit bis zu seiner Auflösung I 933/34

33 Vgl. Protokoll über die Verhandlung des Parteitags der SPD, Weimar Io.-I5.Juni I9I9, hier 406-457. Zitat: Sinzheimer in der Nationalversammlung, 2I.7.I9I9, in: Sten. Berichte (Reichstag), Bd. 328, I748I750.

34 Vgl. Hugo Sinzheimer, Das Rätesystem. Zwei Vorträge zur Einführung in den Rätegedanken. Frankfurt a. M. I9I9.

35 Vgl. die Anlage zum Schreiben des RWiM an den StS der Reichskanzlei, I3.ro.r926, BArch 60I/760, fol. 29-30, Anlage 50-89', hier fol.56-57. Ähnlich die Begründung zum Regierungsentwurf von Art. 34a, abgedruckt bei Hans Schäffer, Der Vorläufige Reichswirtschaftsrat. Kommentar der Verordnung vom 4. Mai I920. München u.a. I920, I67-I77, hier I77.

36 Vgl. AdR, Kabinett Bauer, Bd. I, Nr. Iог: Besprechung zwischen Reichs- und Ländervertretern im Reichswirtschaftsministerium über die Bildung eines vorbereitenden RWR, I I.I I.I9I9, 369-380 und AdR, Kabinett Bauer, Bd. I, Nr. I 3 I, Kabinettssitzung vom I 5.I 2.I 9I 9, 480 Fn. 5: Erlass der VO am 4.5., Inkrafttreten am 7.5., Einberufung zum 7.7.I920. Der Text der VO findet sich u.a. bei Lilla, Reichwirtschaftsrat (wie Anm. I2), I75-I85.

37 Nach der VO I 920 bestand der RWR aus zehn, nach Branchen gegliederten Gruppen: In der Praxis setzte sich jedoch intern bald die Gliederung nach Arbeitgebern, Arbeitnehmern und „beteiligte[n] Volkskreise[n]“ durch, die Sinzheimers Konzept widersprach. Vgl. Lilla, Reichswirtschaftsrat (wie Anm. I2), 35, 39$67,67-86$.

38 Der Gesetzentwurf in Sten. Berichte (Reichstag), Bd. 4I9, Anlage Nr.3709 bzw. Sten. Berichte (Reichs- 
vorläufig; von den Bezirkswirtschaftsräten und den Arbeiterräten hatte man sich bereits lange zuvor verabschiedet. ${ }^{39}$

So unerwartet sein legislatives Scheitern für die Zeitgenossen auch war ${ }^{40}$, so wenig überrascht es aus theoretischer Sicht: Denn grundsätzlich sind Prozessen „expliziten Verfassungswandels“ aufgrund von Pfadabhängigkeiten und sich wechselseitig verstärkenden Wünschen der unterschiedlichen Vetospieler sehr enge Grenzen gesetzt. ${ }^{4 \mathrm{I}}$ Es lohnt daher, auf die Institutionalisierung des RWR „beyond big legislative changes“42 zu blicken. Denn den maßgeblichen Akteuren war rasch klar, dass die ursprünglichen Pläne weder für die Praxis taugten noch (spätestens seit dem Krisenjahr 1923) ernsthaft weiter verfolgt werden sollten. ${ }^{43}$ Die praktische Arbeit wurde ohnehin in den drei Ausschüssen für Wirtschafts-, Sozial- und Finanzpolitik erledigt, die sich ihrerseits wiederum in eine Vielzahl situativ etablierter Arbeits- und Unterausschüsse verzweigten. Der sich während der I92oer Jahre in der täglichen, alles andere als randständigen Praxis entfaltende modus operandi erweist sich so als das Ergebnis eines komplexen Wechselspiels ökonomischer Handlungs-

tag), Bd. 430, Anlage Nr.348. Curtius an Trendelenburg, I 7.9.1927, BArch R 310I/20464, fol.5I-54. Vgl. Der Vorläufige Reichswirtschaftsrat I 927-I 933. Berlin I933, 200-222; Lilla, Reichswirtschaftsrat (wie Anm. I2), 94-I 16; Harry Hauschild, Der vorläufige Reichswirtschaftsrat I920-I926. Denkschrift. Berlin I926, 4955I 8; Franz Hederer, How to Handle Economic Power? Law-Making and the Reich Economic Council in Weimar Germany, in: Management and Organizational History 20I9, DOI: I0.I080/I7449359.20I 9. I659822.

39 Realisiert wurde von Art. I65 WRV letztlich nur die Basisstufe der Arbeiterräte (Betriebsrätegesetz vom 4.2.I920, RGBl. I920, I47-I74) und mit dem „Vorläufigen Reichswirtschaftsrat“ (Verordnung vom 4.5.I920, RGBl. I920, 858-869) die Spitze der Wirtschaftsräte, allerdings mit erheblich limitierten Befugnissen. Vgl. knapp Gerhard Anschütz, Die Verfassung des Deutschen Reichs vom I r.August I9I9. Berlin I933, 748. Zum Ende des RWR vgl. Lilla, Reichswirtschaftsrat (wie Anm. I2), I 20-I 24 und BArch R 3IOI/ 8434 und BArch R 43-I/I I97, fol.92-94.

40 So hatte die Vossische Zeitung am 5.Juli I 930 vermeldet, dass das „Gesetz vom Reichstag beschlossen“ sei.

4I Vgl. Arthur Benz, Das Zusammenspiel der Ebenen beim expliziten und impliziten Verfassungswandel, in: Christoph Hönnige et al. (Hrsg.), Verfassungswandel im Mehrebenensystem. Wiesbaden 20I I, 2 I-40.

42 Jacob S. Hacker/Paul Pierson/Kathleen Thelen, Drift and Conversion: Hidden Faces of Institutional Change, in: James Mahoney/Kathleen Thelen (Eds.), Advances in Comparative-Historical Analysis. Cambridge 20I5, I80-208, Zitat I98-I99. Vgl. auch James Mahoney/Kathleen Thelen, A Theory of Gradual Institutional Change, in: Dies. (Eds.), Explaining Institutional Change. Ambiguity, Agency, and Power. Cambridge $2010, \mathrm{I}-37$.

43 Die Verhandlungen des Verfassungsausschusses des RWR bieten hierzu reichlich Material; vgl. stellvertretend die Begründung des Gesetzentwurfes von 1927 durch Wirtschaftsminister Curtius, I 9.I I.I926, BArch R 40I/569, fol.3. 
zwänge, politischer Präferenzen, praktischer Chancen und nicht zuletzt externer Schocks. ${ }^{44}$

Auffallend ist, wie sehr die Überzeugung, als Ort für Sacharbeit ein Pendant zum „Tohuwabohu“45 im Reichstag zu bilden, alle institutionellen, politischen und ökonomischen Veränderungen überdauerte. Ob die einzelnen Akteure freilich tatsächlich davon überzeugt waren, allein mit ökonomischen Fakten statt politischen Dogmen zu operieren, oder aber ein technokratisches Selbstbild nur inszenierten, ist kaum verlässlich zu beurteilen. ${ }^{46}$ Gleichwohl trug der beständig bekräftigte Anspruch dazu bei, der Praxis des RWR eine „institutionelle DNA“ einzuschreiben, die mehr als bloß rhetorisch wirkte ${ }^{47}$ : Sie fungierte als zentrale Ressource der Selbstvergewisserung und Immunisierung vor allem dann, wenn sich die Mitglieder des RWR (nicht ohne Grund) gezielt von Regierung und Reichstag ignoriert wähnten. ${ }^{48}$ Und so entsprach die Betonung der „Sachlichkeit“ auch einem rationalen Kalkül, auf dieser Ebene einen Raum des offenen und kritischen Diskurses schaffen und sich als eigenständige Institution neben den ökonomischen, technokratischen und politischen Akteuren positionieren zu können. Aus dieser Zwitterstellung erklärt sich sowohl der prekäre Status des RWR als auch der spezifische Mehrwert, den auch Industrievertreter in ihm erkannten und der offenbar nicht über die bekannten Kanäle des Lobbyismus ${ }^{49}$ - Öffentlichkeitsarbeit, direkter Zugang zu Parlament und/

\footnotetext{
44 Vgl. etwa die Beispiele bei Hederer, How to Handle (wie Anm.38). Siehe auch die Arbeit des Zentralausschusses des RWR im Kontext der kontroversen Debatten um „aktive Konjunkturpolitik“ an der Jahreswende I931/32, die in der Forschung bisher nur am Rande wahrgenommen wurden: Bericht des Zentralausschusses, I2.3.I932, BArch R 40I/206, fol.395-399'. Ferner die Tätigkeitsberichte des RWR: Hauschild, Reichswirtschaftsrat (wie Anm. 38), und Der Vorläufige Reichswirtschaftsrat (wie Anm. 38).

45 Max Cohen, Was wird mit dem Reichswirtschaftsrat? In: Sozialistische Monatshefte 36, I930, H. 8, 750-754, hier 754 .

46 Vgl. etwa mit entsprechender Vorsicht Max Hachenburg, Lebenserinnerungen eines Rechtsanwalts. Stuttgart I978, hier I84-206: Hachenburg, angesehener Mannheimer Jurist und Mitglied des RWR von I920-I933, berichtet von seinen persönlichen Erfahrungen, und hebt vor allem die „rein sachliche“ (205) Arbeit des RWR und die Tendenz zum Ausgleich zwischen den Lagern hervor.

47 So etwa der ehemalige Wirtschaftsminister Eduard Hamm im Wirtschaftspolitischen Ausschuss des RWR, 5.8.I930, BArch R 401/407, fol.388: Es sei die vornehmste Aufgabe des RWR, „überstiegene Hoffnungen auf ein gewisses Mass zurückzuführen und gerade von uns aus, die wir nicht politisch die Dinge zu sehen haben, zu sagen, wie komplex die Dinge sind.“

48 Vgl. etwa Max Cohen, 2I. Sitzung des Reparationsausschusses des RWR, 25.I I.I92I, BArch R 40I/6I3, fol. 284-285; Hans Kraemer, ebd.fol.3I 2.

49 Knapp Hederer, How to Handle (wie Anm.38).
} 
oder Regierung - befriedigt werden konnte: die Möglichkeit, am Rande der Öffentlichkeit und ohne politischen Konformitätsdruck kontroverse policies offen zu diskutieren, Netzwerke zu pflegen und politische Entscheidungen zu beeinflussen. Allerdings korrespondierte der Ausformung eines institutionellen Selbstverständnisses eben gerade nicht die Verfestigung verbindlicher Verfahren zu seiner Beteiligung am politischen Entscheidungsprozess. Der RWR avancierte so auf dem Feld der Wirtschafts-, Sozial- und Finanzpolitik zu einem Vetospieler, dessen Position angesichts institutioneller „Eifersüchteleien“, fehlender politischer Instrumente und nicht zuletzt der über allem schwebenden Frage nach dem konkreten Design der Weimarer „Wirtschaftsverfassung“ aber stets dynamisch und prekär blieb. ${ }^{50} \mathrm{Er}$ verkörperte damit einen spezifischen Realtypus der institutionellen Kanalisierung organisierter Wirtschaftsmacht, der einerseits dem offenen Horizont der Zeit nach I9I8 Rechnung trug ${ }^{51}$, andererseits die Labilität der daraus erwachsenden Ordnungsversuche ausdrückte. Diese Ambiguität anzuerkennen, eröffnet Einsichten in die zeitgenössische Wirtschaftsordnung: Denn dass der RWR als Institution zur Ordnungsstiftung durch Versachlichung nach I945 zunächst als Vorbild für einen etwaigen Bundeswirtschaftsrat diente, man sich dann aber für ein gänzlich anderes Modell ökonomischer Politikberatung entschied ${ }^{52}$, verdeutlicht die Dynamik, mit der Wettbewerb, Wirtschaftsmacht und ökonomische Ordnung unter veränderten Bedingungen institutionell kanalisiert und politisch organisiert werden konnten.

50 Siegfried Tschierschky, Wirtschaftsverfassung. Breslau I924; Franz Neumann, Über die Voraussetzungen und den Rechtsbegriff einer Wirtschaftsverfassung, in: Die Arbeit 8, I93 I, H. 6, 588-606. Zitat des Vorsitzenden des RWR, Edler von Braun, im Reparationsausschuss, 28.6.I92I, BArch R 40I/6II, fol.3I5-3I6. Zum Vetospielertheorem vgl. grundlegend George Tsebelis, Veto Players. How Political Institutions Work. Princeton, NJ 2002.

5I Vgl. mit Blick auf die WRV etwa Christoph Gusy, Die Weimarer Verfassung zwischen Überforderung und Herausforderung, in: Der Staat 55, 2016, 29I-3 I 8, hier 305-3 I0; auch Moritz Föllmer et al., Einleitung: Die Kultur der Krise in der Weimarer Republik, in: Moritz Föllmer/Rüdiger Graf (Hrsg.), Die „Krise“ der Weimarer Republik. Zur Kritik eines Deutungsmusters. Frankfurt a. M. 2005, 9-4I, 38.

52 Vgl. Nützenadel, Stunde (wie Anm. I I), I36-I44. 


\section{Ordnung am Markt: Kartelle, Konzentration und Wettbewerb}

Die Ordnung der wirtschaftspolitischen Strukturen war eine Sache, jene der ökonomischen Praxis eine andere, und dies meinte in der Zwischenkriegszeit vor allem Fragen (un-)lauteren Wettbewerbs durch Kartelle und Konzerne. Keines der beiden Phänomene war neu, vielmehr setzten sich Trends fort, die weit bis ins wilhelminische Deutschland zurückreichten. Großindustrielle Komplexe hatten sich insbesondere in den Montan-, Metall- und Elektroindustrien gebildet; seit I904 schlossen sich weite Teile der deutschen Chemiebranche zusammen. ${ }^{53}$ Im Krieg sowie nach I920 beschleunigte sich die Unternehmenskonzentration gewaltig. Vertikale Zusammenschlüsse suchten komplette Verwertungsketten vom Rohstoff bis zum Fertigprodukt zu etablieren, während horizontale Fusionen auf Skaleneffekte durch Kapazitätszusammenlegungen zielten. Beide Trends huldigten dem Zeitgeist der Rationalisierung, versuchten aber zugleich, geeignete Antworten auf die Strukturkrise der Wirtschaft zu liefern. ${ }^{54}$ So erwies sich die von Geiler I 922 diagnostizierte „Kartellmüdigkeit“ und die Verdrängung marktregelnder Organisationen durch Fusionen als trügerisch: Kartelle und Konzerne schlossen einander keineswegs aus, sondern gingen Hand in Hand. Hatte die Zahl der in Deutschland tätigen Produktions-, Preis- und Absatzkartelle vor dem Krieg schon bei rund 600 gelegen, bezifferten Ökonomen und Juristen ihre Menge am Vorabend der Weltwirtschaftskrise auf rund 3000. ${ }^{55}$ Die bekannte Formel, nach der

53 Gottfried Plumpe, Die I. G. Farbenindustrie AG: Wirtschaft, Technik und Politik I 904-I 945. Berlin I990.

54 Geiler, Der genossenschaftliche Gedanke (wie Anm. 24), Io9; zur Verbindung von Rationalisierung und Konzentration siehe auch den späteren Beitrag von Heinrich Kronstein, Konzentration und Technik, in: Die Justiz 6, I930/3I, 3-I9. Zu den Rationalisierungskonzepten der I920er Jahre: Christian Kleinschmidt, Rationalisierung als Unternehmensstrategie. Die Eisen- und Stahlindustrie des Ruhrgebiets zwischen Jahrhundertwende und Weltwirtschaftskrise. Essen I993; Martina Heßler, Kulturgeschichte der Technik. Frankfurt 2012, 38-7I.

55 Zitat: Karl Geiler, Formen der wirtschaftlichen Konzentration [I 922], in: Ders., Gesellschaftliche Organisationsformen, 6I-90, hier 89; vgl. Hartmut Berghoff/Ingo Köhler/Harald Wixforth, Navigation im Meer der Interessen. Binnenwirtschaftspolitische Steuerungsinitiativen des Reichswirtschaftsministeriums, in: Holtfrerich (Hrsg.), Reichswirtschaftsministerium (wie Anm. I6), 42 I-5I6, hier 494f. Etwas niedrigere Zahlen bei Jeffrey Fear, Cartels, in: Geoffrey Jones/Jonathan Zeitlin(Eds.), The Oxford Handbook of Business History. Oxford 2008, 268-292, hier 275, und Harm Schröter, International Cartels, in: Joel Mokyr (Ed.), The Oxford Encyclopedia of Economic History. Vol.3. Oxford 2003, I23-I26, hier I 25 f. 
Deutschland „the classical land of cartels“ war, schien I93 I den Nagel auf den Kopf zu treffen. ${ }^{56}$

Doch die tiefgreifende Krise unterstrich zugleich, dass das Stabilitätsversprechen von Kartellen und Konzernen nicht für bare Münze zu nehmen war. Hatten bereits während der Hyperinflationsjahre Kritiker die Unternehmensfusionen als spekulative, mithin unverdiente Manöver attackiert und Kartelle für Versorgungsengpässe verantwortlich gemacht, so gewannen derart normative Erwägungen während der tiefen Depression noch an Gewicht: Der Preis der Wettbewerbsbeschränkung war nicht nur hoch, er blieb nun auch ohne Gegenwert. Derartige Vorwürfe verbanden sich besonders mit Kartellen, deren „organisierte Intransparenz“57, wiewohl nicht immer richtig verstanden, Grund zum Anstoß gab und die im Verdacht standen, Kosten auf die Verbraucher abzuwälzen. Die Fehlerdiagnose war somit nicht länger nur theoretischer und ideologischer Natur, sondern stellte auf praktische Unzulänglichkeiten ab..$^{8}$

Die Kontroverse um die ökonomische und ethische Bewertung marktregelnder Verbände reichte dabei bis ins Kaiserreich zurück. Schon I 894 hatte sich der Verein für Socialpolitik auf seiner Jahrestagung mit dem Thema beschäftigt, und die berühmte Entscheidung des Reichsgerichts drei Jahre später, die Kartellverträge gerichtlich durchsetzbar machte, hatte die Debatte eher befeuert denn beigelegt. Zwei Juristentage I902 und I904 sowie die Kartellenquête des Reichstags blieben ihrem Tenor nach zwar überwiegend kartellfreundlich, konnten jedoch nicht kaschieren, dass sich die Kartelle und ihre Befürworter in der Bringschuld befanden: Wettbewerbsbeschränkungen blieben dank einer vokalen, kritischen Minderheitsposition sowie wiederkehrender Konjunkturkrisen immer erklärungs- und verteidigungsbedürftig. ${ }^{59}$

56 Louis Domeratzky, Cartels and the Business Crisis, in: Foreign Affairs Io, I93 I, 34-53, hier 37.

57 Eva-Maria Roelevink, Organisierte Intransparenz. Das Kohlensyndikat und der niederländische Markt I9I5-I932. München 20I5.

58 Siehe die Überschau bei Berghoff/Köhler/Wixforth, Navigation, 489-504.

59 Vgl. Nörr, Leiden (wie Anm. I3), I f., I 8-24, Piepenbrock, Gedanke (wie Anm. 23), I 9 I, und Hans Pohl, Die Entwicklung der Kartelle in Deutschland und die Diskussion im Verein für Socialpolitik, in: Helmut Coing/ Wilhelm Walter (Hrsg.), Wissenschaft und Kodifikation des Privatrechts im I 9. Jahrhundert, Bd. 4. Frankfurt a. M. I979, 203-235, hier 225-233; Bernhard Großfeld, Die Kartellrechtsdiskussion vor dem Ersten Weltkrieg, in: ebd.279-289. Siehe auch Dieter Krüger, Nationalökonomen im wilhelminischen Deutschland. Göttingen I982, 74-IOI. 
In den frühen Weimarer Jahren - und dies motivierte wohl auch Geilers Beobachtung - schien es kurzzeitig, als stünde eine Trendwende bevor, als das Reichswirtschaftsministerium die Initiative für eine I923 im Wege der Ermächtigungsgesetzgebung erlassene „Verordnung gegen Mißbrauch wirtschaftlicher Machtstellungen“ ergriff, um den Wettbewerb einer staatlichen Kontrolle zu unterwerfen. Tatsächlich aber stand die „Kartellverordnung“ schon ihren Prämissen nach auf tönernen Füßen und war wohl eher taktischen Erwägungen geschuldet, sah sich das Ressort doch genötigt, „Ersatz zu schaffen für ein Kartellgesetz, d.h. Parlament und öffentlicher Meinung teilweise nachzugeben. “60 Die geringe Eigenmotivation spiegelten vage Formulierungen, stumpfe Instrumente und ein Grundmuster, das eher auf bargaining denn auf Sanktionen setzte. Statt die Kartellpolitik auf eine neue Grundlage zu stellen, hinterließ die Verordnung den „Eindruck der Halbherzigkeit und Unentschlossenheit“, der statistisch noch untermauert wurde. Die Zahl der Kartellverträge nahm drastisch zu, und einzelne Branchen wie die Stahl- und Kaliindustrien waren nahezu lückenlos in marktregelnden Organisationen erfasst; I930 waren rund 40 Prozent der gesamten deutschen Industrieproduktion kartelliert. ${ }^{6 \text { I }}$ Auch neuerlich eingeholte wissenschaftliche Expertise brachte zwar manch neue Erkenntnis, änderte aber wenig an der politischen Ausrichtung. Sowohl im Zuge einer Wirtschaftsenquête, deren Abschlussberichte 1930 vorgelegt wurde, als auch auf dem Deutschen Juristentag I 928 in Salzburg dominierten die Kartellbefürworter. ${ }^{62}$ Am Ende konstatierte Hugo Sinzheimer, der selber keine eindeutige Position zum Thema vertrat, weil er in Kartell- und Koalitionsfreiheit verwandte Rechtsgüter erblickte, die genuin liberalen und den Wettbewerb priorisierenden Stimmen seien klar in der Minderheit geblieben. ${ }^{63}$

\footnotetext{
60 RGBl. I923, I067-I071; Zitat: Wirtschaftsministerium, Vortragsskizze zur „Wirtschaftspolitik gegenüber den Kartellen“, I 2.6.1922, BArch R 310I/20892, fol.398.

6I Berghoff/Köhler/Wixforth, Navigation (wie Anm. 55), 496ff., Zitat 498; Nörr, Leiden (wie Anm. I3), 4962; Hartmut Bechtold, Die Kartellierung der deutschen Volkswirtschaft und die sozialdemokratische Theorie-Diskussion vor 1933. Frankfurt a. M. 1986, 242.

62 Vgl. Verhandlungen und Berichte des Unterausschusses für allgemeine Wirtschaftsstruktur (I. Unterausschuß), 3. Arbeitsgruppe: Wandlungen in den wirtschaftlichen Organisationsformen, 4. Teil: Kartellpolitik, 2 Bde. Berlin 1930, und Verhandlungen des Fünfunddreißigsten Deutschen Juristentages (Salzburg), 3 Bde. Berlin/Leipzig I 928/1929.

63 Hugo Sinzheimer, Der 35. Deutsche Juristentag in Salzburg, in: Die Justiz 4, H. I, I928/29, 95-98; vgl. auch den Bericht von Oswald Lehnich, Der gegenwärtige Stand der Kartellfrage, in: Zeitschrift für die gesamte Staatswissenschaft 87, 1929, 50I-544.
} 
Angesichts eines von politischen Erwägungen geformten Rahmens lag es nahe, dass die Rechtsprechung zur Konkretisierung aufgerufen war. Ein besonders instruktives Bespiel für die rechtliche Formung einer politischen Frage stellt der sogenannte „Benrather Tankstellenfall“ dar ${ }^{64}$ : Am I 8. Dezember I93 I entschied der II. Zivilsenat des Reichsgerichts letztinstanzlich die Klage des Tankstellenbesitzers Hubert Engels aus Düsseldorf-Benrath, der sich gegen die Preispolitik einer sogenannten ,Konvention` zu wehren suchte. In diesem Syndikat hatten sich einige große Mineralölkonzerne im Oktober I928 zusammengeschlossen, um „die abträglichen Folgen des gegenseitigen Wettbewerbs tunlichst einzuschränken und die von ihnen ins Leben gerufene Verkaufsorganisation zu festigen." 65 Engels hatte sein Benzin von einem nicht der Konvention angehörenden Unternehmen bezogen und konsequent unter dem Preis des Syndikats angeboten; dieses wiederum reagierte prompt und wies seine Mitglieder in Benrath an, ihrerseits stets um einen Pfennig günstiger zu verkaufen. Mit der Behauptung, das Syndikat beabsichtige „seine wirtschaftliche Vernichtung“, klagte der Tankstellenbesitzer gegen diese Praxis - und bekam Recht. $^{66}$

Der unscheinbare Fall führte zu einer wegweisenden juristischen Klärung der Frage, welche Formen „lauterer“ Wettbewerb unter den Bedingungen einer kartellierten Wirtschaft annehmen durfte und welche als sittenwidrig zu verwerfen waren. Mit Rudolf Callmann, Rudolf Isay und Hans Carl Nipperdey waren im Verfahren drei der namhaftesten Wettbewerbsrechtler der Zeit mit einschlägigen, einhellig kartellfreundlichen Gutachten an die Öffentlichkeit getreten. Im Kern wurden anhand des Falls ethische Prämissen einer „guten“ Ordnung am Markt verhandelt, die sich zwar eindeutiger rechtlicher Subsumption entzogen, gleichwohl aber rechtlich formuliert werden wollten. Als juristisches Problem schälte sich dabei das Spannungsverhältnis zwischen Wettbewerb und (der damit möglicherweise bewirkten) „Vernichtung“ des Konkurrenten heraus: Während Isay und vor allem Callmann dazu tendierten, diese als übliches „Ziel des kaufmännischen Wettbewerbs“ zu qua-

64 Vgl. Nörr, Leiden (wie Anm. I3), hier 95-Io0; Nörr, Zwischen den Mühlsteinen (wie Anm. I3), I43I6r. Zum Gegenstand des Verfahrens vgl. II. Zivilsenat, Urteil vom I8. Dezember I93I, in: RGZ I34, 342356. Die RGZ enthalten allerdings nur eine gekürzte Fassung des Urteils; ungekürzt und nicht zur Veröffentlichung bestimmt findet es sich in den Kartellakten des Justizministeriums: BArch R 300r/79r6, fol. $345-354^{\circ}$.

65 BArch R 300I/79I6, fol. I45'-I46.

66 BArch R 300I/79I6, fol. I46؛. 
lifizieren ${ }^{67}$, schlug Nipperdey eine Differenzierung vor, die schließlich vom Gericht übernommen wurde - allerdings mit gegensätzlicher Stoßrichtung: Nipperdey unterschied zwei Arten von Wettbewerbshandlungen, nämlich „solche, die in der Ausdehnung des eigenen Geschäftsbetriebs, genauer in der Förderung der Absatztätigkeit mit den Mitteln der eigenen Leistung bestehen“, und andere, „die nur eine Behinderung des Mitbewerbers herbeiführen“ würden. Auch wenn es im „Wirtschaftskampf“ kein objektives Recht auf wirtschaftliche Existenz gebe, sei ein „Vernichtungszweck“ dann als „unsittlich“ zu klassifizieren, „wenn er Selbstzweck“ sei. ${ }^{68}$

Die vermeintlich luzide Unterscheidung zwischen „Leistungswettbewerb“, als dessen mögliche Folge die Existenzvernichtung grundsätzlich in Kauf zu nehmen war, und „Behinderungswettbewerb“, der (nur) dann gegen die guten Sitten verstieß, wenn er allein auf Vernichtung der Konkurrenz zielte ${ }^{69}$, verschob das Problem aber nur. Denn die Entscheidung, ob nun das Verhalten eines Marktakteurs diesen Zweck verfolgte oder ein nichtintendiertes Ergebnis lauterer Geschäftspraktiken darstellte, wurde somit der Rechtsprechung überlassen. Und während Nipperdey in seinem Rechtsgutachten die Preisunterbietungspolitik des Syndikats als Leistungswettbewerb einstufte, erschien dem Gericht diese Argumentation als „nicht recht verständlich“, ja „verfehlt“. Ganz eindeutig handele sich hier „um einen Fall schwersten Behinderungswettbewerbs“, der aufgrund des offensichtlichen „Vernichtungszwecks“ sittenwidrig sei. ${ }^{70}$ Die Rechtsprechung dürfe nicht nur den zunehmenden Konzentrationsprozess der Wirtschaft in Rechnung stellen. Vielmehr sei es in Zeiten wie diesen schlicht ein „Unding“, wenn Unternehmen die Preise ausgerechnet für einen „lebensnotwendigen Bedarfsgegenstand“ wie Kraftstoff künstlich in die Höhe trieben. Auch aus diesem (im Kern freilich hochpolitischen) Grund sei im Sinne des Klägers zu entscheiden. ${ }^{71}$

Der Streitfall zwischen Außenseiter und Syndikat dokumentiert, dass und wie um 1930 vor dem Hintergrund der schwelenden Kartellproblematik und des tiefen Konjunktureinbruchs Vorstellungen legitimen Wettbewerbs rechtlich neu profi-

67 Rudolf Callmann, Außenseiter und Kampfpreise, in: Juristische Wochenschrift I930, I647-I650, hier 1647 .

68 Hans Carl Nipperdey, Wettbewerb und Existenzvernichtung, in: Kartell-Rundschau 28, I930, I27-I52, hier I40, I3I, I36.

69 Ebd. I40ff.

70 Zitate: BArch R 300I/70I6, fol. I54, I5I', I52', I53.

7I Zitate: BArch R 300I/70I6, fol. I53, I53“. 
liert wurden und ungeachtet ihrer juristischen Formung einer höchst umstrittenen politisch-ethischen Agenda verpflichtet waren. Das von Nipperdey eingeführte Kriterium, die Lauterkeit einer Wettbewerbshandlung von der ihr zugrunde liegenden Intention abhängig zu machen, bot da nur scheinbar eine Lösung: Denn die Frage konnte abhängig von ideologischem Standpunkt, ethischen Überzeugungen und politischen Rahmenbedingungen durchaus unterschiedlich beantwortet werden. Hier wird das zeitgenössisch intensiv diskutierte und vor allem in Bereich des Wirtschaftsrechts virulente Problem eines zunehmenden Rekurses auf Generalklauseln greifbar, welche Flexibilität und Anpassungsfähigkeit suggerierten, diese aber mit potenzieller Rechtsunsicherheit erkauften. ${ }^{72}$ Trotz aller Bemühungen um rechtliche Erfassung und damit um normative Rationalisierung blieb die Frage wirtschaftlicher Ordnung also eminent politisch und kam an sozial-moralischen Prämissen über das Verhältnis von Eigeninteresse und Gemeinwohl, individuellem Verhalten und nichtintentionalen Handlungsfolgen nicht vorbei. Sie in rechtliches Vokabular zu kleiden versprach allein noch keine inhaltliche Sicherheit.

Die späten I92oer Jahre markierten mithin auf unterschiedlichen Ebenen einen Paradigmenwechsel, auch wenn dieser zunächst nur geringe Sichtbarkeit aufwies. Denn während berühmte Namen wie Joseph Schumpeter es vermieden, sich eindeutig zu positionieren und sich stattdessen mit ambivalenten Einschätzungen begnügten, die „fast schon den Charakter einer Absolution“ für die kartellpolitischen Versäumnisse der Vergangenheit hatten ${ }^{73}$, hoben sich zwei jüngere Köpfe - Franz Böhm und Heinrich Kronstein - mit klaren, teils scharfen Worten von der herrschenden Lehre ab. Kronstein hatte zunächst bei Geiler studiert und I 924 bei Martin Wolff in Berlin promoviert, ehe er eine Doppelkarriere als Wirtschaftsanwalt und Dozent in Heidelberg einschlug. ${ }^{74}$ Böhm hatte seine Staatsexamen in Freiburg i. Br. abgelegt, um dann ins Reichswirtschaftsministerium zu wechseln, wo er unter der Leitung von Ministerialrat Paul Josten in der Kartellabteilung tätig war. I93 I kehrte Böhm in den Breisgau zurück und wurde dort mit einer zweiteiligen wettbewerbs-

72 In diesem Kontext auch die Rechtsprechung des Reichsgerichts der I $920 e r$ Jahre zur kriegsbedingten Leistungsstörung und zur Aufwertungsproblematik: RGZ Ioo, I 29 (I92 I); RGZ I03, 328 (I922); RGZ I07, 78 (I923); RGZ I07, 370 (I 924); RGZ I I I, 320 (I 925). Grundlegend Justus Wilhelm Hedemann, Die Flucht in die Generalklauseln. Eine Gefahr für Recht und Staat. Tübingen I933.

73 Berghoff/Köhler/Wixforth, Navigation (wie Anm. 55), 503.

74 Heinrich Kronstein, Die abhängige juristische Person. München I93I; vgl. Zacher, Entstehung (wie Anm. 25), I6of. 
rechtlichen Arbeit in den beiden folgenden Jahren erst promoviert und dann habilitiert. $^{75}$

Kronstein und Böhm sammelten in ihrer praktischen Arbeit Erfahrungen mit jenen ordnungspolitischen Fragen, die im Mittelpunkt ihrer lebenslangen wissenschaftlichen Interessen stehen sollten - und keinem der beiden gefiel, was er sah. In der „Justiz“, dem von Sinzheimer und Radbruch mitherausgegebenen Organ des Republikanischen Richterbundes, erschienen I 928-29 gleich drei Artikel aus ihrer Feder. Darin unterstrichen Böhm wie Kronstein, dass wirtschaftliche Freiheit längst nicht mehr allein vom Staate, sondern mindestens ebenso, wenn nicht gar mehr durch die Akkumulation privater Macht bedroht werde. Private, so argumentierte Kronstein, schufen reale Machtbeziehungen, die über Interessengemeinschaftsund Kartellverträge, Absatz- und Konzernbeziehungen rechtliche Bindungswirkung beanspruchten. Dass der Staat in dieses Machtgefälle weder eingreifen konnte noch wollte, es sogar protegierte, erkannten beide Juristen als Problem. Entsprechend hielt Böhm die Neigung seiner Kollegen, unternehmerische Freiheit auch zur Entäußerung derselben nutzen zu können, für eine Travestie liberaler Errungenschaften. ${ }^{76}$

Mit ihrer grundlegenden Kritik am wettbewerbspolitischen Status quo fanden sich Böhm und Kronstein, beide bürgerlich-liberale Geister mit sozialem Verantwortungsgefühl im Sinne Geilers, in einer bemerkenswerten Situation. Während sie unter den zeitgenössischen Wirtschaftsjuristen klar in der Minderheit waren, erhielten sie Zuspruch von den linksliberalen und sozialdemokratischen Kollegen, denen die „Justiz“ ein Forum bot ${ }^{77}$, ohne dass sie freilich mit den reformmarxistischen

75 Biographische Informationen bei Eckard Rehbinder, Heinrich Kronstein (I897-I972), in: Bernhard Diestelkamp/Michael Stolleis (Hrsg.), Juristen an der Universität Frankfurt am Main. Baden-Baden I989, 252-267, und Rudolf Wiethölter, Franz Böhm (I895-I977), in: ebd.208-25I, sowie David Gerber, Heinrich Kronstein and the Development of United States Antitrust Law, in: Marcus Lutter/Ernst C. Stiefel/Michael H. Hoeflich (Hrsg.), Der Einfluss deutscher Remigranten auf die Rechtsentwicklung in den USA und in Deutschland. Tübingen I993, I53-I69.

76 Böhm, Problem (wie Anm. I5), 324-345. Heinrich Kronstein, Wirtschaftsrecht - Rechtsdisziplin und Zweig der Rechtstatsachenkunde, in: Die Justiz 3, I927/28, 2 I5-225; ders., Zum Problem: Staat und Wirtschaftsmacht. Bemerkungen anlässlich der Großbankenfusion, in: Die Justiz 5, I929/30, I37-I46. Vgl. Zacher, Entstehung (wie Anm. 25), 240-244, und Rehbinder, Kronstein (wie Anm. 75), 263 f.

77 Siehe etwa Franz Neumann, Recht und Eisenkampf, in: Die Justiz 4, I928, H. 2, I I3-I 2 I; vgl. Lehnich, Stand (wie Anm. 63), 52 I f., 53 I, 534-536, und Heinrich Kronstein, Briefe an einen jungen Deutschen. 2.Aufl. München I968, I45. 
Zielen etwa Franz Neumanns einverstanden waren. Ebenso wenig teilten Kronstein und Böhm die antirepublikanischen Affekte zur Rechten, stimmten aber dessen ungeachtet mit mancher Krisendiagnose Carl Schmitts überein, etwa der Beobachtung, dass mächtige Interessengruppen den Staat zu zerstören drohten. ${ }^{78}$ Böhm fand sich zudem in der Gesellschaft Ernst Forsthoffs, wenn er dezisionistisch vortrug, die einmal beschlossene (Wirtschafts-)Verfassung könne nicht einfachrechtlich oder gar privatim ausgehöhlt und ad absurdum geführt werden. ${ }^{79}$ Doch während Forsthoff und Schmitt daraus auf die Unzulänglichkeit demokratischer Organisation schlossen und dem kommenden autoritären, gar „totalen“ ${ }^{80}$ Staat das Wort redeten, hielt Kronstein eben diese Folgerung für einen kategorialen Irrtum. Statt einen einfachen Primat zu fordern, legte er „ein[en] Ausgleich [...] zwischen den beiden gesellschaftlichen Organisationen“ (Staat und Privatwirtschaft; Anm. der Verf.) nahe. Mit dem Primat des Staates konnte er nichts anfangen; „die Lehre vom souveränen Staat führt nicht zu ihm“, notierte er I929 nüchtern. ${ }^{8}$ Noch stimmte dem auch Franz Böhm zu.

\section{Ordnung erzwingen? „Wirtschaftsstaat“ und „totaler“ Staat}

Die Frage, wie die Veränderungen im Verhältnis von Staat und Wirtschaft zu erfassen und die daraus resultierenden Probleme praktisch zu lösen seien, verdichtete sich in den frühen I 93oer Jahre vor allem auf die Marktmacht privater Wirtschaftsakteure. Die Debatte um „Deutschland als Wirtschaftsstaat“ ist dabei vor allem deshalb aufschlussreich, weil sich darin nicht nur die latente Distanz des (ökonomischen) Liberalismus zur parlamentarischen Demokratie manifestierte. ${ }^{82}$ Vielmehr

78 Kronstein, Problem (wie Anm. 76), I4 I f; Eucken, Strukturwandlungen (wie Anm. I4), 307; Schmitts erste Fassung erschien I929 als Aufsatz: Carl Schmitt, Der Hüter der Verfassung, in: Archiv des öffentlichen Rechts 55, I929, Nr.2, I6I-237.

79 Korrespondenz Forsthoff-Schmitt, 23.I., 8.4. und 7.5.1932, in: Dorothee Mußgnug/Reinhard Mußgnug/Angela Reinthal (Hrsg.), Briefwechsel Ernst Forsthoff Carl Schmitt (I 926-I974). Berlin 2007, 39-42. Die Überlegungen Böhms und Schmitts gegenüberstellend: Wiethölter, Böhm (wie Anm. 75), $230 f f$. Vgl. Sean Irving, Limiting Democracy and Framing the Economy. Hayek, Schmitt and Ordoliberalism, in: History of European Ideas 44, 2018, I I3-I 27.

80 Ernst Forsthoff, Der totale Staat. Hamburg 1933.

8I Kronstein, Problem (wie Anm.76), I45.

82 Prägend Huber, Wirtschaftsstaat (wie Anm. I4). Vgl. jüngst auch Hacke, Existenzkrise (wie Anm.7), $335-366$. 
ist es die These grundsätzlich inkompatibler Funktionslogiken von Ökonomie und Politik, die den in der WRV zumindest angedeuteten Anspruch zur - wie auch immer verstandenen - „Demokratisierung“ der Wirtschaft durch die Partizipation der Beteiligten als störend bzw. systemwidrig ausschloss und daher nach anderen, radikaleren Lösungen verlangte. ${ }^{83}$

Diese für liberale Autoren zunächst irritierenden Annahmen mit der „unübersichtlichen Lage“ in den frühen I93oer Jahren zu erklären, erscheint aber unbefriedigend, zumal eine Kohärenz des Denkens unterstellt wird, die aus ideengeschichtlicher Perspektive grundsätzlich problematisch ist. ${ }^{84}$ Fruchtbarer ist es, diese Texte als einen Beitrag zur hochumstrittenen Frage nach den Konturen der (zukünftigen) Wirtschaftsordnung zu lesen, ob „liberal“ oder nicht. ${ }^{85}$ Auffallend ist, dass ganz unterschiedliche Stimmen weitgehend konvergente Diagnosen stellten: In der historisch gewachsenen und kaum mehr zu entwirrenden Verknotung von Ökonomie, Politik und Staat saß demnach letzterer nur mehr scheinbar am längeren Hebel, während in Wahrheit längst die Wirtschaft das Ruder übernommen hatte. ${ }^{86}$ Wollte man nicht dem wahrgenommenen Trend der zunehmenden Vermischung von privater und öffentlicher, wirtschaftlicher und staatlicher Sphäre tatenlos zusehen oder diese gar forcieren, sei eine durchgreifende „Entpolitisierung“ ${ }^{87}$ nötig, die nicht

83 So etwa Fritz Naphtali u. a., Wirtschaftsdemokratie. Ihr Wesen, Weg und Ziel. Berlin I928. Das Problem der „Demokratisierung durch Beteiligung“ war (und blieb) selbst in sozialdemokratischen Kreisen höchst umstritten. Vgl. Hacke, Existenzkrise (wie Anm. 7), 360 ff., sowie exemplarisch Neumann, Voraussetzungen (wie Anm.50).

84 Zitat: Hacke, Existenzkrise (wie Anm. 7), 357. Vgl. die Kritik an ideengeschichtlicher Mythologisierung bei Quentin Skinner, Meaning and Understanding in the History of Ideas, in: H \& T 8, I 969, 3-53. Siehe auch Franz Hederer, Im Sog des Egalitären. Ökonomisches Denken und der Wandel gesellschaftlicher Ordnungsvorstellungen in der Sattelzeit. Göttingen 20I4, 29-40. Hacke, Existenzkrise (wie Anm.7), 340, 355 ff., betont zwar die Heterogenität einzelner Autoren, hält an ihrer Kohärenz als Gruppe der „späteren Ordoliberalen“ (ebd.356) allerdings fest. Vgl. Jan-Otmar Hesse, Wirtschaft als Wissenschaft. Die Volkswirtschaftslehre in der frühen Bundesrepublik. Frankfurt a. M. 20 o.

85 Aus dem politischen Spektrum etwa Edgar Tatarin-Tarnheyden, Kopfzahldemokratie, organische Demokratie und Oberhausproblem, in: Zeitschrift für Politik I5, I 926, 97-I22; besonders explizit Max Frauendorfer, Der ständische Gedanke im Nationalsozialismus. München I932. Vgl. auch Ernst Fraenkel, Kollektive Demokratie, in: Die Gesellschaft 6, I929, H. 8, I03-I I 8; Hermann Heller, Autoritärer Liberalismus? In: Ders., Gesammelte Schriften. Zweiter Band: Recht, Staat, Macht. Leiden I 97 I, 643-653.

86 Eucken, Strukturwandlungen (wie Anm. I4), 303.

87 Der Begriff ist weder historisch stabil noch analytisch unproblematisch. Hierzu exemplarisch Moritz Julius Bonn, Die Auflösung des modernen Staates (I92 I), in: Ders., Zur Krise der Demokratie. Politische Schriften in der Weimarer Republik I9I9-I932. Berlin 20I5, I I I-I36, hier I 29, I30. 
nur bezweckte, staatliche Eingriffe in die Wirtschaft zu reduzieren oder gar zu eliminieren, sondern vielmehr auf eine grundsätzliche Sphärentrennung des Politischen vom Ökonomischen zielte: So kritisierte etwa Ernst Rudolf Huber die „planlose“ Unordnung des Marktes und beschwor eine pouvoir neutre, die sich dem ökonomischem Wettbewerb enthalten und dadurch Ordnung schaffen sollte, während Eucken die durch Marktabsprachen bequem gewordenen Akteure durch „die Peitsche der Konkurrenz" zu disziplinieren trachtete. ${ }^{88}$

Das klang zunächst vertraut, doch besaß das Programm der „Entpolitisierung“ noch eine weitere Dimension, die weniger darauf zielte, einen weitgehend freien Markt zu schaffen, als vielmehr den als schädlichen wahrgenommenen „Einfluss der Massen“ einzudämmen. ${ }^{89}$ Die greifbare Skepsis gegenüber der Abhängigkeit (wirtschafts-)politischen Handelns von demokratischer Legitimation drängt den Vergleich zu Schmitt geradezu auf, der in der Kolonisierung des Staates durch Private eine logische Konsequenz des liberalen, grundsätzlich „unpolitischen“ Parlamentarismus erkannte. Schmitts Forderung nach Entökonomisierung richtete sich denn auch darauf, die bloß „quantitative Totalität“ des Staates auf ihren qualitativen Kern, d.h. die Fähigkeit zur politischen Entscheidung, zu konzentrieren. Das Programm der ordoliberalen „Entstaatlichung“ wich davon nur scheinbar ab: Im Vordergrund stand die Absicht, die öffentliche Hand als Wirtschaftsakteur aus dem Spiel zu nehmen und zugleich mit einer rahmengebenden Wächterrolle zu betrauen. ${ }^{\circ} \mathrm{Ob}$ dies nun die Wiederherstellung des Primats der Politik über die Wirtschaft oder aber umgekehrt nur die Etablierung eines Staates „unter der Aufsicht des Marktes“ zur Folge haben sollte, mag diskutabel sein. ${ }^{9 \mathrm{I}}$ Greifbar wird allerdings, dass die ordoliberale

88 Huber, Wirtschaftsstaat (wie Anm. I4), 2 I, 29; Eucken, Strukturwandlungen (wie Anm. I4), 298; Hacke, Existenzkrise (wie Anm. 7), 360.

89 Eucken, Strukturwandlungen (wie Anm. I4), 3I8, mit Carl Schmitt, Weiterentwicklung des totalen Staats in Deutschland (Januar I933), in: Ders., Positionen und Begriffe im Kampf mit Weimar - Genf - Versailles I923-I939. Hamburg I940, I85-I90, hier I87. Dass beide Autoren damit durchaus Unterschiedliches implizierten, steht außer Frage.

90 Carl Schmitt, Die Wendung zum totalen Staat (I93I), in: Ders., Positionen und Begriffe im Kampf mit Weimar - Genf - Versailles I 923-I939. Hamburg I940, I 46-I 57, hier I 54; Schmitt, Neutralisierungen (wie Anm. I4). Zum Begriff der „Totalität“ Schmitt, Weiterentwicklung (wie Anm. 89), I86f. Auch Eucken, Strukturwandlungen (wie Anm. I4), 3I9; Ernst Rudolf Huber, Selbstverwaltung der Wirtschaft, in: Deutsches Volkstum I932, H. 2, 883-889, hier 885; Heinrich Göppert, Staat und Wirtschaft, in: Deutsche Juristen-Zeitung 38, 1933, Sp.48-52. Anders aber Hacke, Existenzkrise (wie Anm. 7), 347.

9I Vgl. Michel Foucault, Geschichte der Gouvernementalität II. Die Geburt der Biopolitik. Vorlesungen am 
Agenda von Entflechtung und Wettbewerb ein ökonomisches Ordnungsmodell semantisch zu etablieren suchte, an dem sich staatliches Handeln auszurichten hatte. Anders als das Reichsgericht, das in seiner Entscheidung zum Benrather Tankstellenfall nur eine alternative Interpretation angeboten hatte, wurde damit die Wirtschaftsordnung als Ganzes in Frage gestellt und für einen „Kapitalismus in neuartiger Gestalt“ (Eucken) plädiert. ${ }^{92}$

Euckens Überlegungen sind in der Rückschau oft verklärt und sein tief verankerter, nicht selten ins Reaktionäre reichender Kulturkonservatismus gegen seine zu einem „umfassenden bürgerlich-liberalen Denken“ tendierenden politischen Neigungen aufgerechnet worden. ${ }^{93}$ Die von ihm herausgegebene Zeitschrift „Die Tatwelt“ (mit der bekannteren jungkonservativen Monatsschrift „Die Tat“ gänzlich unverbunden, aber nicht immer uneins) ist als quasi-oppositionelles Organ gewürdigt worden, das liberalen, regimekritischen Gedanken nach 1933 einen geschützten Raum gegeben habe. Dies ist nicht ganz falsch - aber eben auch nur die halbe Wahrheit. Schon vor dem nationalsozialistischen Machtantritt vertraten die Autoren in Euckens Zeitschrift rechtskonservative Gedanken, bezogen außenpolitisch revisionistische Positionen und fanden für die Republik selten gute Worte. ${ }^{94}$

Auch Franz Böhms Beiträge zur „Tatwelt“ wurden retrospektiv - unter anderem von Edith Eucken-Erdsiek, Philosophin und nach 1950 Nestorin der ordoliberalen Schule - entsprechend gewürdigt. Insbesondere der „Recht und Macht“ betitelte, I934 in zwei Tranchen veröffentlichte Beitrag aus Böhms Feder firmierte hier prominent. ${ }^{95}$ Gemeinsam mit der 1933 erschienenen Monographie „Wettbewerb und

Collège de France I978-I 979. Frankfurt 2006, Zitat I68. Hierzu auch Hacke, Existenzkrise (wie Anm. 7), 335338, und Jan-Otmar Hesse, „Der Mensch des Unternehmens und der Produktion“. Foucaults Sicht auf den Ordoliberalismus und die „Soziale Marktwirtschaft“, in: Zeithistorische Forschungen 3, 2006, 29I-296.

92 Eucken, Strukturwandlungen (wie Anm. I4), 3 I 8.

93 So jüngst Hacke, Existenzkrise (wie Anm. 7), 350. Anders die ältere Darstellung von Dieter Haselbach, Autoritärer Liberalismus und soziale Marktwirtschaft. Baden-Baden I991, sowie Ralf Ptak, Vom Ordoliberalismus zur Sozialen Marktwirtschaft: Stationen des Neoliberalismus in Deutschland. Opladen 2004.

94 Vgl. Joshua Rahtz, The Politics of Order: Ordo-Liberalism from the Inter-War Period through the Long I970s, Phil. Diss UCLA 20I7, sowie Uwe Dathe/Nils Goldschmidt, Wie der Vater, so der Sohn? Neuere Erkenntnisse zu Walter Euckens Leben und Werk anhand des Nachlasses von Rudolf Eucken in Jena, in: ORDO 54, 2003, 49-74, hier 59-63.

95 Franz Böhm, Recht und Macht, in: Die Tatwelt Io, I934, I I5-I32, I69-I93; Edith Eucken-Erdsiek, Franz Böhm in seinen Anfängen, in: Heinz Sauermann/Ernst-Joachim Mestmäcker (Hrsg.), Wirtschaftsordnung und Staatsverfassung. Fschr. für Franz Böhm zum 80. Geburtstag. Tübingen I975, 9-I4, hier I I. Vgl. auch ihr Editorial zur Erstausgabe von Ordo: Chaos und Stagnation, in: Ordo I, I948, 3-I5, hier I3. 
Monopolkampf“ legte Böhm just in jenem Jahr, in dem das NS-Regime die Macht übernahm und konsolidierte, seine systematischen Überlegungen zur Wirtschaftsverfassung vor. Als „unzeitgemäße Betrachtung“ hat sein Schüler Ernst-Joachim Mestmäcker die Schrift eingeordnet: Böhm habe seinerzeit die Nationalsozialisten mit liberalen Ideen konfrontiert, die den ihren „direkt entgegengesetzt“ gewesen seien. ${ }^{96}$ Nun hätten die Nationalsozialisten für einen solchen Gegensatz erst einmal ein stringentes wirtschaftspolitisches Programm haben müssen ${ }^{97}$, und da ein solches fehlte, erzeugte Böhms Buch kaum Anstoß. Huber spottete zwar, sein Freiburger Kollege habe die nationalsozialistische Revolution nicht recht verstanden und argumentiere noch immer auf dem Boden der Weimarer Verfassung - allerdings konnte man dasselbe mit Fug und Recht über Hubers eigene, fast zeitgleich veröffentliche Habilitationsschrift sagen. Tatsächlich begannen Böhms Konflikte mit Regimevertretern erst in der zweiten Hälfte der I930er Jahre und entzündeten sich was für den Juristen sprach - eher an den rassistischen als den wirtschaftlichen Zielen des NS-Staates. ${ }^{98}$

In Letzteren zeigte sich Böhm schon an der Schwelle zur Diktatur durchaus aufgeschlossen gegenüber einer entschlossen regulierenden Regierung. Sicher: nicht alles in seinen I933-34 publizierten Schriften konnte dem neuen Regime schmecken. Der Spott auf Kosten „nationalpädagogischer Wirtschaftsmoralisten“ und Zweifel daran, wie effektiv eine „noch so diktatorisch bevollmächtigte Staatsaufsicht“ am

96 Ernst-Joachim Mestmäcker, Einführung, in: Böhm, Wettbewerb und Monopolkampf (wie Anm. I5), 5I4, hier 5. Vgl. die Skizze bei Hans Zacher, Aufgaben einer Theorie der Wirtschaftsverfassung, in: Helmut Coing/Heinrich Kronstein/Ernst-Joachim Mestmäcker (Hrsg.), Wirtschaftsordnung und Rechtsordnung. Fschr. zum 70. Geburtstag von Franz Böhm am I6. Febr. I965. Karlsruhe I965. 63-109, besonders 65-67.

97 Dazu LudolfHerbst, Gab es ein nationalsozialistisches Wirtschaftssystem? In: Albrecht Ritschl (Hrsg.), Das Reichswirtschaftsministerium in der NS-Zeit. Wirtschaftsordnung und Verbrechenskomplex. (Wirtschaftspolitik in Deutschland I9I7-I990, Bd. 2.) Berlin 2016, 622-644; Stephen G. Gross, The Nazi Economy, in: Shelley Baranowski/Armin Nolzen/Claus-Christian W. Szejnmann (Eds.), A Companion to Nazi Germany. Hoboken 2018, 265-279.

98 Ernst Rudolf Huber, Rezension, in: Juristische Wochenschrift I7, I934, I038-1039, nach Nörr, Leiden (wie Anm. I3), Ior. Eben dies wurde an Hubers Buch (Ernst Rudolf Huber, Wirtschaftsverwaltungsrecht. Institutionen des öffentlichen Arbeits- und Unternehmensrechts. Tübingen I932) beanstandet, vgl. Johannes Bähr, „Recht der staatlich organisierten Wirtschaft“. Ordnungsvorstellungen und Wandel der deutschen Wirtschaftsrechtslehre im „Dritten Reich“, in: Ders./Ralf Banken (Hrsg.), Wirtschaftssteuerung durch Recht im Nationalsozialismus. Studien zur Entwicklung des Wirtschaftsrechts im Interventionsstaat des „Dritten Reichs“. Frankfurt 2006, 445-472, hier 455. Zu Böhms Karriere in den I93oern vgl. Tamara Zieschang, Das Staatsbild Franz Böhms. Stuttgart 2003. 
Ende zu sein vermöge, waren dazu ebenso wenig angetan wie die Prämisse, Freiheit sei für alle „große[n] Aufgaben von schöpferischen Persönlichkeiten“ unabdingbar. ${ }^{99}$ Doch gerade in der Konzeptualisierung von Freiheit zeigte sich, dass Böhms liberale Prinzipien flexibel waren. In Abgrenzung zu klassischen Positionen des ökonomischen wie des politischen Liberalismus erkannte Böhm in der Freiheit keinen Selbstzweck: Freiheit sollte zur Ordnung führen, das Recht war Mittel zum Zweck. Wie autoritär diese Ordnung verstanden wurde, drückte sich in der Idee der „Herrschaftsverfassung“ aus: Wo immer der Markt nicht im Gemeinschaftssinne operierte, sollte der Staat, der dessen Inhalt definierte, eingreifen. Für Böhm löste sich der vermeintliche Widerspruch von Freiheit und Ordnung damit auf: Erst Ordnung erlaubte wirtschaftliche Freiheit, die sich im „abstrakte[n] Verfahren“ des Marktes entfaltete. Und seiner Natur nach „Kampf“ befand sich der Markt im Einklang mit den Ordnungsvorstellungen des neuen Regimes. ${ }^{\text {Ioo }}$

Böhms Ausführungen stellten keine opportunistische Wende dar, sondern gründeten in seinen tief verwurzelten Zweifeln an privatwirtschaftlicher Macht und dem Wunsch, ein starker Staat möge das Heft endlich in die Hand nehmen. Und zumindest in ordnungspolitischer Hinsicht schien das erste Jahr der NS-Herrschaft Anlass zur Hoffnung zu geben. Hier war eine Regierung, die entschlossen, ja rücksichtlos handelte, um die - von ihr selbst definierten - Interessen der Volksgemeinschaft durchzusetzen. Böhm konnten die sprachlichen und semantischen Parallelen zu seinem eigenem Begriffsapparat kaum entgehen, wenn er den zurückliegenden „Entartungs- und Verfälschungsprozess des Rechtes der freien Wirtschaft“ attackierte, den „Auslesekampf“ des Wettbewerbs lobte und die „Züchtung wirtschaftsberuflicher Höchstleistungen“ forderte. In moralisierender Diktion, die „den Sieg des Schlechteren über den Besseren“ ablehnte, bediente Böhm gängige, nicht nur von der NS-Propaganda genutzte und regelmäßig antisemitisch aufgeladene Klischees vom „gewissenlosen Spekulanten [und] des Schiebers“. ${ }^{\text {IoI }}$ Böhms Schrift war kein nationalsozi-

99 Böhm, Wettbewerb und Monopolkampf (wie Anm. I5), 238; ders., Recht und Macht (wie Anm.95), I 83. Ioo Böhm, Recht und Macht (wie Anm. 95), I70-I 75, I 83-I87; ders., Wettbewerb (wie Anm. I 5), I07, I I 8I 2 I, I47. Vgl. Haselbach, Autoritärer Liberalismus (wie Anm.93), und Hacke, Existenzkrise (wie Anm.7), 365 f.

Ior Böhm, Wettbewerb (wie Anm. I5), I7, 9I, I I 8, I 20, I49. Zu den ökonomischen Spielarten antisemitischer Stereotypen vgl. Saul Friedländer, Nazi Germany and the Jews. The Years of Persecution, I933-I939. New York I997, 65, und Martin H. Geyer, Verkehrte Welt: Revolution, Inflation und Moderne. München I9I4-I924. Göttingen I998, I86, 244, 280, 385 f. 
alistischer Programmentwurf, jedoch mit dem Regime dort kompatibel, wo dieses willens und fähig schien, mit der Anarchie privater Egoismen aufzuräumen, ohne den unternehmerischen Impuls zugunsten einer Zentralplanungswirtschaft abzuschaffen. ${ }^{\text {I02 }}$

Dieses Einschätzung drückte sich auch in der Initiative aus, die Böhm mit dem Freiburger Fakultätskollegen Hans Großmann-Doerth und dem ebenfalls im Breisgau lehrenden Eucken (von dem das Begriffspaar freie Verkehrswirtschaft/Zentralplanungswirtschaft stammte) in den folgenden Jahren ergriff. ${ }^{103}$ Dass die Mitte der I93oer Jahre noch namenlose Schule damit keineswegs nur in der badischen Provinz ihrer subversiven Wege ging, sondern mit Sendungsbewusstsein versuchte, Einfluss zu nehmen, ist inzwischen bekannt ${ }^{\text {I04 }}$, obschon die Bewertungen zwischen ideologischer Übereinstimmung und technokratischer „Manipulationsabsicht“ erheblich differieren. ${ }^{\text {I05 }}$ Die Hochschullehrer um Eucken und Böhm brachten keine Sympathie für die Massenpartei NSDAP und insbesondere nicht für deren rassistische Politik auf, doch widersprach dies schwerlich ihrem Anliegen, das systemische Vakuum der NS-Wirtschaftpolitik zu füllen. Ihren programmatischen Anspruch formulierten Böhm, Eucken und Großmann-Doerth in der I936/37 lancierten Reihe „Ordnung der Wirtschaft“, namentlich im gemeinsamen Vorwort des ersten Bandes. ${ }^{\text {I06 }}$ Mit „Unsere Aufgabe“ betitelt, war der dreizehn Seiten lange Text mehr Ma-

I02 Zugleich unterschied sich Böhm deutlich etwa von Armin Müller-Armacks Nähe zum NS-Regime; vgl. Ptak, Ordoliberalismus (wie Anm.93), 8I, 98, und Haselbach, Autoritärer Liberalismus (wie Anm.93), 88-92, I 22.

I03 Alexander Hollerbach, Jurisprudenz in Freiburg. Beiträge zur Geschichte der Rechtswissenschaftlichen Fakultät der Albert-Ludwigs-Universität. Tübingen 2007, 299f. Vgl. Köster, Wissenschaft (wie Anm. 9), $30 \mathrm{If}$.

I04 Die ältere Sicht bei Wiethölter, Böhm (wie Anm.75), 229; Nörr, Leiden (wie Anm. I3), ror; David Gerber, Constitutionalizing the Economy. German Neo-Liberalism, Competition Law and the "New Europe", in: American Journal of Comparative Law 42, I 994, 25-84, hier 30. Eine revidierte Perspektive, vor allem mit Blick auf den von Josten im Reichswirtschaftsministerium mit initiierten Professorenausschuss: Daniela Rüther, Freiburger Nationalökonomen auf dem Weg in den Widerstand: Neue Erkenntnisse über die Rolle des ,Professorenausschusses‘ von I939, in: Historisch-Politische Mitteilungen I0, 2003, 75-94, besonders $77 \mathrm{f} ., 83 \mathrm{f}$.

I05 Erstere Lesart vor allem bei Haselbach, Autoritärer Liberalismus (wie Anm. 93), 77-95, letztere unter anderem bei Rüther, Freiburger Nationalökonomen (wie Anm. I04), 8I und ähnlich überhöhenden Darstellungen der Freiburger Schule.

I06 Da Böhms Band erst später in Druck ging, wurde das Vorwort auch der Studie F.A. Lutz' vorangestellt; Friedrich August Lutz, Das Grundproblem der Geldverfassung. Stuttgart I936; Hans Gestrich, Neue Kreditpolitik. Stuttgart I936; Franz Böhm, Die Ordnung der Wirtschaft als geschichtliche Aufgabe und rechtsschöp- 
nifest denn Editorial und wischte mit wuchtigen Formulierungen überkommene Traditionen beiseite, zuvorderst die beiden historischen Schulen in Rechts- und Wirtschaftswissenschaften. Dagegen setzten die drei Freiburger in dezidiert Schmitt'schen Begriffen die Wirtschaftsverfassung „als eine politische Gesamtentscheidung über die Ordnung des nationalen Wirtschaftslebens“. ${ }^{\text {107 }}$

In seiner eigenen Monographie ging Böhm noch einen Schritt weiter: Das Programm der Schriftenreihe basiere auf der Grundannahme, „daß die staatliche Wirtschaftspolitik das wirtschaftliche Geschehen geistig und machtmäßig in den Griff bekommt. Das ist aber nur möglich, wenn die Wirtschaft durchsichtig und streng geordnet ist und wenn diese Ordnung [...] von der Nation geistig erfaßt und erlebt und von den wirtschaftenden Volksgenossen mit Hingabe und Disziplin befolgt wird. “ ${ }^{08}$ Sprachlich wie inhaltlich war dies ein Angebot an das Regime, und die folgenden 200 Seiten unterstrichen dies nachdrücklich. Einschübe wie die Forderung, wirtschaftliches Handeln am theoretischen Ideal auszurichten, statt das Faktische zur Norm zu heben - Letzteres beschrieb die NS-Wirtschaftspolitik recht gut -, übten allenfalls zurückhaltende Kritik. ${ }^{\text {I09 }}$

Schwerer wogen die Zugeständnisse gegenüber staatlicher Regulierung in Landwirtschaft und Arbeitsrecht, beim dogmatisch kaum zu verteidigenden Vierjahresplan und im autoritären Geist, der dem Buch innewohnte. So qualifizierte Böhm Verbraucher danach, ob ihr Konsum „zur Lebens- und Kraftsteigerung der Gesamtwirtschaft“ beitrug oder als „[g]esundheitsschädlicher, kulturwidriger, die Sittlichkeit gefährdender [...] Bedarf“" zu bekämpfen war. Grundsätzlich stipulierte der Jurist, „[n] ur soweit der Wettbewerb zur Ordnung führt, kann private wirtschaftliche Freiheit gewährt werden; soweit dies nicht der Fall ist, darf die Wirtschaft nicht frei sein. “ ${ }^{\text {IIo }}$ Ordnung konnte und musste erzwungen werden. ${ }^{\text {III }}$

\footnotetext{
ferische Leistung. Stuttgart I937; Leonhard Miksch, Wettbewerb als Aufgabe. Die Grundsätze einer Wettbewerbsordnung. Stuttgart 1937.

I07 Franz Böhm/Walter Eucken/Hans Großmann-Doerth, Unsere Aufgabe, in: Böhm, Ordnung (wie Anm. I06), viii-xxi, Zitate ix, xii, xix. Vgl. Haselbach, Autoritärer Liberalismus (wie Anm.93), 84f., und Ptak, Ordoliberalismus (wie Anm.93), 90-93.

Io8 Böhm, Ordnung (wie Anm. I06).

Io9 Ebd.50, 66.

I Io Ebd.77-80, 88ff., I08, II I.

I I Insofern war es nicht ohne Ironie, dass ein marxistisch geschulter Beobachter wie Neumann im Monopolkapitalismus, organisiert in Konzernen und Kartellen, einen der Pfeiler des NS-Staats erkannte, während Böhm hoffte, dass der noch namenlose Behemoth die Hydra bändigen möge. Böhm war näher an Ernst
} 
Die ökonomische Praxis des Dritten Reiches wurde diesen Erwartungen kaum gerecht, private Macht verschwand keineswegs, und das „Faustrecht des Monopolkampfs“ blieb nicht nur bestehen, sondern gewann bis Kriegsende noch an Drastik. ${ }^{\text {II2 }}$ Doch verdross dies Eucken und Böhm offenbar nicht so sehr, als dass sie nicht weiter ihre Ideen angeboten hätten, zumal diese dank terminologischer Konvergenzen unter regimenahen Juristen anschlussfähig waren. Die offensichtlichen Diskrepanzen zu ihren Überlegungen rationalisierten die Freiburger als temporäre, aufrüstungs- und kriegsbedingte Ausnahmen. Und nicht zuletzt konnte es nicht schaden, weiter für die eigene Theorie zu werben, wie etwa auf der bekannten, unter dem Dach der Akademie für Deutsches Recht veranstalteten Berliner Tagung I 942 - in einer wahrscheinlicher werdenden nicht nationalsozialistischen Zukunft mochte sich dies womöglich auszahlen. ${ }^{\text {II3 }}$

\section{Endlich Ordnung? Markt und Wettbewerb vor der Bundesrepublik}

Im Sommer I945 war diese Zukunft da, und nach den chaotischen Wochen, die auf die Kapitulation folgten, (re-)konstituierten sich rasch konservativ-liberale, oft christlich getönte Netzwerke, in denen auch die mit Markt und Macht hantierenden Juristen und Ökonomen wie Böhm, Eucken und Walter Hallstein, aber auch Forsthoff, vertreten waren. ${ }^{\text {II }}$ Hier wurden die konzeptionellen Trockenübungen der letzten Kriegsjahre fortgesetzt, bot sich doch im Neuanfang die Gelegenheit, das Verhält-

Fraenkels (ebenfalls noch unpubliziertem) Befund, dass der Doppelstaat legale wie gewaltsame Optionen bot, die kapitalistischen Interessen von Stabilität und Berechenbarkeit dienten; vgl. Meierhenrich, Remnants (wie Anm. 26), 29f., I96f.

I 2 Ebd.I48, I50. Zu Konzentration und Zwangskartellierung siehe Dieter Swatek, Unternehmenskonzentration als Ergebnis und Mittel nationalsozialistischer Wirtschaftspolitik. Berlin I 972.

I 3 Die Beiträge unter anderem von Böhm, Eucken und Miksch sind gesammelt bei Günter Schmölders (Hrsg.), Der Wettbewerb als Mittel volkswirtschaftlicher Leistungssteigerung und Leistungsauslese. Berlin I942. Vgl. Haselbach, Autoritärer Liberalismus (wie Anm.93), 94-99; Jan-Otmar Hesse, Zur Semantik von Wirtschaftsordnung und Wettbewerb in nationalökonomischen Lehrbüchern der Zeit des Nationalsozialismus, in: Bähr/Banken (Hrsg.), Wirtschaftssteuerung (wie Anm.98), 473-508, $494 \mathrm{ff}$.

I 4 Böhm war Mitglied der im Sommer 1945 begründeten Christlichen Arbeitsgemeinschaft, während Hallstein und Forsthoff der I948 ins Leben gerufenen Mundus Christianus angehörten; vgl. Meinel, Forsthoff (wie Anm. 26), 308; Walter Otto Ötsch/Stephan Pühringer/Katrin Hirte, Netzwerke des Marktes. Ordoliberalismus als Politische Ökonomie. Wiesbaden 2018, I52; Michael Klein, Westdeutscher Protestantismus 
nis von Staat und Wirtschaft auf eine einheitliche, theoretisch begründete Basis zu stellen. Böhm etwa musste seine früheren Überlegungen nur gelinde neu justieren, um sie in den neuen alten Rahmen der freiheitlichen Demokratie und des Rechtstaats einzupassen. Im Einklang mit Eucken und Wilhelm Röpke, vor allem aber mit seinem rechtswissenschaftlichen Kollegen Hallstein, der liberales Denken nicht mehr als Freiheit vom Recht, sondern „Freiheit im Recht“ programmatisch definierte ${ }^{\text {II5 }}$, suchte Böhm, den Widerspruch zwischen individueller Freiheit und staatlicher Regulierung in gewohnter Weise zu überbrücken, indem er die öffentliche Hand zum Garanten ihrer unsichtbaren Entsprechung auf der Markseite machte. Neu war indes der Akzent auf den ,sozialen Staat', den Böhm ab I 945 legte, nicht zuletzt, um sozialdemokratische Umverteilungsforderungen zu kontern. Die Maxime der „sozialen Gerechtigkeit“ erschien prominent in liberalkonservativen Nachkriegsdokumenten, während das zuvor eingeübte Vokabular von Auslese und Kampf wieder auf Wettbewerb und Prozesshaftigkeit heruntergekocht wurde. Dem „tyrannischen“ Staat erteilten die Freiburger und ihre Mitstreiter eine Absage. Aber dass es „der straffsten Staatsaufsicht“ bedurfte, um individuelle Grundrechte „auch gegen private Machtträger“ zu sichern, daran ließen sie nach wie vor keinen Zweifel. ${ }^{\text {I } 6}$

Mit dem I948 folgenden Launch der Zeitschrift „Ordo“ sollte die deutsche Variante neoliberalen Denkens sich selbst ihren Namen geben. Früher im Druck und einflussreicher indes war die „Süddeutsche Juristen-Zeitung“ (SJZ). Unter der Herausgeberschaft von Geiler, Hallstein, Radbruch und Hans Ehard versammelten sich hier zwischen I 946 und I950 nicht nur klangvolle Namen der deutschen Rechtswissenschaft; die SJZ avancierte zu einem der zentralen Foren, in denen die staats- und wirtschaftsrechtlichen Grundzüge der Bundesrepublik verhandelt wurden. Insbesondere Böhms Artikel - namentlich seine Auseinandersetzung mit dem Sozialdemokraten Adolf Arndt - gaben die Richtung vor. Unter den wenigen zurückkehrenden Emigranten, die in der SJZ Platz fanden, ihre Erfahrungen und Meinungen in die

und politische Parteien. Anti-Parteien-Mentalität und parteipolitisches Engagement von I 945 bis I 963 . Tübingen 2005, I65, I82.

I 5 Walter Hallstein, Wiederherstellung des Privatrechts, in: Süddeutsche Juristen-Zeitung I, I946, I-7, Zitat 6.

I 6 Franz Böhm, Die Bedeutung der Wirtschaftsordnung für die politische Verfassung, in: Süddeutsche Juristen-Zeitung I, I 946, I 4 I-I 49, Zitat I42; ders., Kartellauflösung und Konzernentflechtung Spezialistenaufgabe oder Schicksalsfrage? In: Süddeutsche Juristen-Zeitung 2, I947, 495-506, Zitat 502. Vgl. Klein, Westdeutscher Protestantismus (wie Anm. I I4), I 65 ff. 
juristische Debatte einzubringen, war derweil Heinrich Kronstein. ${ }^{117}$ Wie viele seiner Kollegen vor der nationalsozialistischen Verfolgung geflohen, hatte er seine Karriere in den USA mit Zweitstudium und -promotion neu begonnen, aber sein Interesse an kartell- und konzernrechtlichen Fragen bewahrt. Angesichts des wachsenden Bedarfs nach solcher Expertise, den die amerikanischen enemy studies besonders nach I94 I entwickelt hatten, war Kronstein zu einem gefragten, wenngleich gelegentlich missverstandenen Fachmann für die deutsche Wirtschaftsorganisation aufgestiegen. ${ }^{\text {II } 8}$

In eben dieser Funktion kehrte der badische Jurist I 945 in das „Land [s]einer ersten Liebe“ zurück. Skeptisch gegenüber allen Ansätzen einer strafenden Besatzungspolitik suchte Kronstein nach vertrauenswürdigen Partnern für die Alliierten - und fand Hallstein und Böhm. Während Hallstein ein Bekannter aus gemeinsamen Tagen an Martin Wolffs Lehrstuhl war und im Februar I 946 das Rektorat der Frankfurter Universität übernahm, offenbarte sich der Kindheitsfreund Böhm als ordnungspolitischer Bruder im Geiste: „Franz ist wunderbar“, notierte Kronstein im Oktober I945. ${ }^{\text {II } 9}$ Fortan sollte der Emigrant entscheidend dazu beitragen, Böhms Prestige als „real hero of the anti-Hitler fight“ und „excellent leader of the German anti-trust movement“ bei den US-Behörden zu stärken. Zugleich zählte der inzwischen zum Katholizismus konvertierte Kronstein zu jenen, die - wie auch Hallstein und Ehard bei der SJZ - die Kluft zwischen den christlichen Konfessionen überbrücken halfen, indem er den christlich-sozialen Flügel als Partner im Kampf gegen Kartelle und Konzerne empfahl. Dass Kronstein größere Bereitschaft zeigte, staatliche Eingriffe in das Marktgeschehen zu rechtfertigen, und das Wirtschaftsrecht seit langem als „soziales Recht“ verstand (was sich auch in seiner anhaltenden Sympathie für Sinzheimer ausdrückte), war vor dem Hintergrund des liberalen Legitimitätsdefizits der Nachkriegsjahre nur hilfreich. ${ }^{120}$

II7 Heinrich Kronstein, Der Amerikanische Richter im Wirtschaftsrecht, in: Süddeutsche Juristen-Zeitung 5, I950, 79I/792-795/796. Friedrich Darmstädter, auch er ein Weimarer Wettbewerbsrechtsexperte, fand sich ebenfalls unter den Beiträgern: ders., Recht und Jurist, in: Süddeutsche Juristen-Zeitung 3, I 948 , $429 / 430-435 / 436$.

I 8 Zu seiner Karriere im Krieg s. Ernst C. Stiefel/Frank Mecklenburg, Deutsche Juristen im amerikanischen Exil (I933-I950). Tübingen I99I, 60f., I36f.; vgl. Gerber, Kronstein (wie Anm. 75), und Priemel, Searching (wie Anm. Io).

I 9 Kronstein, Briefe (wie Anm.77), 42, $249 \mathrm{f}$.

I20 Zitate: [Fragment] Section 2. Cartels and Combines (Decentralization of Economic Structure), no date [I945], und Report on Conditions in Germany industry, [I945], beide in: International Law Institute (ILI), 
Während sich das akademisch-politische Netzwerk zwischen Kronstein, Böhm und Hallstein in den folgenden Jahren verfestigte ${ }^{\mathrm{I} 2 \mathrm{I}}$, stieg die politische Nachfrage nach ihren wettbewerbsrechtlichen Konzepten. Als I947 unter dem Vorsitz Paul Jostens eine Sachverständigenkommission einberufen wurde, um einen Kartellgesetzentwurf für die Zukunft auszuarbeiten, übernahm Jostens früherer Mitarbeiter Böhm die konzeptionelle Führung; Kronstein wurde als informeller Diskutant in die Beratungen einbezogen. Der sogenannte Josten-Entwurf artikulierte dabei beider Juristen Überzeugung, dass private Macht eine mindestens ebenso große Gefahr darstellte wie ihr öffentliches Pendant, und dass nur ein - selber rechtlich eingehegt - starker Staat in der Lage war, die „Monopolbekämpfung[,] eine der wichtigsten

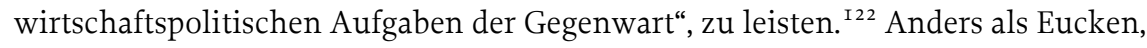
der seinerseits in der französischen Besatzungszone als Berater aktiv war, forderten Böhm und Kronstein ein absolutes Kartellverbot sowie eine scharfe Konzentrationskontrolle mit praktischen Eingriffsrechten durch eine regierungsunabhängige Behörde. ${ }^{\text {I23 }}$

Erfolgreich sollten sie am Ende nur mit Abstrichen sein. Sah es I949 noch nach

Heinrich Kronstein Papers, http://www.ili.org/I 29-about/about/528-heinrich-kronstein-documents-andpublications-archive.html; vgl. Heinrich Kronstein, Wirtschaftsrechtliche Lehren des Falles „Frankfurter Allgemeine Versicherungs-AG“, in: Die Justiz V, I929/30, H. 2, I09-I I4, I Io; Kronstein, Briefe (wie Anm. 77), I 45 ff. In den I 96oer Jahren sollte dies indes zum Auseinanderdriften der liberalen Spielarten Kronsteins und Böhms führen; vgl. Louis Pahlow, Die Entzauberung des Ordoliberalismus, in: Ioo Jahre Rechtswissenschaft in Frankfurt. Erfahrungen, Herausforderungen, Erwartungen. Frankfurt a. M. 20I4, 395-408, hier 403-407.

I 2 I Hallstein berief erst Böhm nach Frankfurt (und später in die Delegation, die das Luxemburger Abkommen mit Israel aushandelte), holte dann Kronstein als Gastprofessor, und dieser folgte ihm schließlich auf seinem Lehrstuhl nach; Kronstein wiederum lud Hallstein und Böhm sowie gemeinsame Schüler wie Kurt Biedenkopf und Ernst Mestmäcker in die USA ein; vgl. Kronstein, Briefe (wie Anm. 77), 249-254; Rehbinder, Kronstein (wie Anm.75), 257f., sowie Kurt Biedenkopf, Heinrich Kronstein, in: Stefan Grundmann/ Karl Riesenhuber (Hrsg.), Deutschsprachige Zivilrechtslehrer des 20. Jahrhunderts in Berichten ihrer Schüler, Bd. I. Berlin 2007, I87-205; Constantin Goschler, Wiedergutmachung: Westdeutschland und die Verfolgten des Nationalsozialismus (I945-I954). München I992, 268-270.

I 22 Schreiben an Böhm, Josten, Otto Veit, Nell-Breuning, H. Ilau, Köppels, Heimerich u.a., 22.6.I 949; Erhard an Kronstein, I6.9.I957; Zitat: Gutachten des Wissenschaftlichen Beirats bei der Verwaltung für Wirtschaft, 24.7.I949; Lecture „German Cartels“, American Foreign Law Association, I957, alle ILI, Kronstein Papers.

I23 Jan-Otmar Hesse, Wissenschaftliche Beratung der Wirtschaftspolitik. Das Bundeswirtschaftsministerium und die Volkswirtschaftslehre, in: Werner Abelhauser (Hrsg.), Das Bundeswirtschafsministerium in der Ära der Sozialen Marktwirtschaft. Wirtschaftspolitik in Deutschland I9I7-I990, Bd. 4. Berlin 20I6, 390-48I, hier 438f. 
einem fundamentalen Kurswechsel aus, der sich auch im Einschwenken Nipperdeys ausdrückte ${ }^{124}$, wurden ihre Vorschläge in der fast ein Jahrzehnt währenden, durch massive Lobbybemühungen deutscher Unternehmensvertreter gekennzeichneten Debatte signifikant abgeschwächt; das I957 schließlich erlassene Gesetz gegen Wettbewerbsbeschränkung sah Ausnahmen vom Kartellverbot vor und ließ der Unternehmenskonzentration beträchtliche Spielräume. Gleichwohl fand sich mit dem Bundeskartellamt eine Behörde berufen, den Markt zu beobachten und das Spiel der Kräfte zugunsten von mehr Freiheit einzuschränken - und mit der Kommission der Europäischen Wirtschaftsgemeinschaft sollte im folgenden Jahr ein zusätzlicher, mächtiger Protagonist hinzukommen. Kronstein, Böhm und Hallstein, letzterer erster Kommissionspräsident, erblickten darin eine durchaus wünschenswerte Praxis: Wenn die kartellfreundliche nationale Praxis unausrottbar war, mochte die wachsende Verflechtung deutschen und europäischen Rechts einen gangbaren Ausweg bieten und der wettbewerbsrechtlich starke Staat dann eben in Brüssel sitzen. ${ }^{\text {I25 }}$

\section{Normative Zentrierung oder: Wie man lernt, Kontingenz zu tolerieren}

Was Juristen wie Nipperdey, Kronstein und Böhm von Nachkriegszeit zu Nachkriegszeit umtrieb, war keineswegs ein marginales Thema nur für Spezialisten. Wie weit unternehmerische Freiheit reichte, ob es eine Freiheit zur Unfreiheit geben

I24 Explizit in Hans Carl Nipperdey, Die Grundprinzipien des Wirtschaftsverfassungsrechts, in: Deutsche Rechts-Zeitschrift 5, I950, I93-I98. Nipperdey hatte sich seit den frühen I930er Jahren kritisch mit dem Behinderungswettbewerb auseinandergesetzt; grundsätzlichere Fragen nahm er nach I 949 vor allem in der Debatte um den Verfassungscharakter des Sozialstaats auf; vgl. Achim Seifert, das Bundesarbeitsgericht in der „Ära Nipperdey“, in: Christian Fischer/Walter Pauly (Hrsg.), Höchstrichterliche Rechtsprechung in der frühen Bundesrepublik. Tübingen 2015, 247-262.

I 25 Lecture „German Cartels“, American Foreign Law Association, I957, ILI, Kronstein Papers, und Heinrich Kronstein, 'Cartels' under the new German Cartel Statute, in: Vanderbilt Law Review I I, I 958, 27 I-30I. Zu Hallstein vgl. Morten Rasmussen, Revolutionizing European Law. A History of the Van Gend en Loos Judgement, in: International Journal of Constitutional Law I2, 20I4, I36-I63. Für die ordoliberalen Positionen zur europäischen Regulierung vgl. Philip Plickert, Wandlungen des Neoliberalismus. Eine Studie zu Entwicklung und Ausstrahlung der „Mont Pèlerin Society“. Stuttgart 2008, 247-250, und Zieschang, Staatsbild (wie Anm. 98), 93 f., 2 I 8f. 
konnte, in welchem Verhältnis staatliche und private Macht zueinanderstanden und welche moralischen Standards „lauterem“ Wettbewerb unterlegt werden sollten, zählte über drei Jahrzehnte zu den Kernfragen gesellschaftlicher Ordnung in Deutschland. Die Debatte begann bereits vor dem Ersten Weltkrieg und blieb aufgrund der letztlich ungeklärten konkreten wirtschaftlichen Verfasstheit erst Weimars, dann des „Dritten Reiches“, schließlich der aus dessen Trümmern entstehenden Bundesrepublik zwischen r9 8 und I948 virulent. Und da sich die Startbedingungen nach dem Zweiten Weltkrieg erheblich günstiger darstellten als knapp 30 Jahre zuvor, schien es den beteiligten Akteuren nicht nur möglich, sondern dringend geboten, die als überfällig wahrgenommenen Richtungsentscheidungen endlich zu treffen. Die Rigidität und Unerbittlichkeit, mit der die Auseinandersetzung über drei Jahrzehnte geführt wurde, speiste sich ganz erheblich aus dieser geteilten Überzeugung. Dass Wettbewerbsfragen unterhalb der Abstraktionshöhen verfassungsrechtlicher Grundsatzdebatten von Carl Schmitt, Hans Kelsen oder Rudolf Smend verliefen (wiewohl sie deren Konzepte oft mit verhandelten), unterstreicht ihre Bedeutung als Untersuchungsgegenstand, ging es doch um die konkrete Ausgestaltung dessen, was Friedrich Glum I 934 als „Wirtschaftsstaatsrecht“ bezeichnete. ${ }^{\text {I26 }}$

Der permanente Schwebezustand der „Wirtschaftsverfassung“ korrelierte mit einer umso stärker ausgeprägten Suche nach Eindeutigkeit und einem kohärenten Prinzip, das die ökonomischen Beziehungen im Innersten zusammenhielt, ohne durch die zentrifugalen Wirkungen des wirtschaftlichen Alltages fortwährend infrage gestellt zu werden. Entsprechend verließ die Debatte um die richtige Distanz zwischen Wirtschaft und Staat rasch die radikalen, oft im Rechts-links-Schema formulierten Streitpunkte um Räte, Sozialisierung, Stände oder schrankenlose Unternehmerfreiheit. Bei allen Unterschieden (nicht zuletzt mit Blick darauf, wie viel Autoritarismus sie vertrugen) befanden sich Böhm, Kronstein und Nipperdey auf der Suche nach einem „soziale[n] Dispositiv des Kontingenzmanagements“, das auf absolute Lösungen verzichtete und stattdessen, wiederum in unterschiedlichem Maße, „Kontingenztoleranz“, mithin Beweglichkeit und Anpassungsfähigkeit bot. ${ }^{\mathrm{I} 27}$

I26 Der Anlass war Hubers Antrittsvorlesung; Glum, Rez. Huber (wie Anm. I7), I I5. Ähnlich auch Fritz Haußmann, Rechtsstaat und „Wirtschaftslenkung“. Ein Beitrag zum Problem: Staat, Recht und Wirtschaft. Basel I938, I I 4.

I27 Vgl. dazu Michael Makropoulos, Tendenzen der Zwanziger Jahre. Zum Diskurs der Klassischen Moderne in Deutschland, in: Deutsche Zeitschrift für Philosophie 39, I99I, 675-687, hier 686. 
Wie liberal - und in welcher Spielart - diese Überlegungen schlussendlich waren und damit zum ideellen Traditionsbestand der Bundesrepublik taugen, mag daher auf den zweiten Blick weniger spannend sein als der Umstand, dass sich die Wege dieser recht buntscheckigen Liberalen immer wieder mit jenen sozialdemokratischer (Sinzheimer, Neumann) oder autoritär gesinnter Geister (Huber, Forsthoff) kreuzten, und dabei keineswegs nur Widersprüche erzeugten. Gemeinsam war der ungleichen Reisegesellschaft von Rechtswissenschaftlern nicht zuletzt, dass sie die Wirtschaftsordnung eher zu reformieren als zu revolutionieren gedachten.

Diese Debatten lassen sich, ein durch den Kirchenhistoriker Berndt Hamm für die Frühe Neuzeit geprägtes Konzept aufnehmend, als Prozess der „,normativen Zentrierung als Intention und Wirklichkeit“ beschreiben - und dies nicht nur wegen jener Parallelen, die sich mit Blick auf Krisenwahrnehmungen und den Hang zu militanten Freund-Feind-Unterscheidungen aufdrängen. Der durch zwei Nachkriegszeiten gerahmte Zeitraum von I9I 8 bis I948 erscheint dann als integrale Phase, in der sich ein Bedarf an „neue[r] Sicherheit und Legitimierung, Klarheit und Ordnung“ verdichtete. ${ }^{\text {I28 }}$ Ansätze, derartige Bedürfnisse zu befriedigen, gab es zuhauf, und sie blieben keineswegs nur Theorie; viele waren jedoch ideologisch umstritten, manche erwiesen sich bald als unpraktikabel, unrealistisch oder überholt. Und auch der Ordoliberalismus, dessen Stunde in der Praxis erst nach I945 schlagen sollte, stand I932 keineswegs bereits parat. Vielmehr bedurfte es der Desavouierung seiner eigenen autoritären Züge und der Einbettung in übernationale epistemische (und nicht zuletzt eminent praktische) Netzwerke, um mit einem anschlussfähigen Vokabular zur rechten Zeit am rechten Ort - zu Beginn des Wiederaufbaubooms - wieder aufzutauchen: als Resultat eines normativen Zentrierungsprozesses, der das in der ersten Hälfte des 20.Jahrhunderts hart umkämpfte Feld wirtschaftlicher Ordnungskonzepte semantisch wie politisch zu bündeln vermochte - und dafür schon bald verklärt wurde.

I28 Berndt Hamm, Normative Zentrierung im I5. und I6.Jahrhundert. Beobachtungen zu Religiosität, Theologie und Ikonologie, in: ZHF 26, I999, I63-202, hier I67 ff. 


\section{Zusammenfassung}

Die erste Hälfte des 20.Jahrhunderts ist gerade in Deutschland von markanten politischen und ideologischen Brüchen gekennzeichnet, die sich tief in das kollektive Gedächtnis eingegraben haben, ohne aber unsere Wahrnehmung von Ökonomie und Staat im gleichen Zeitraum nachhaltig zu prägen. In vorliegendem Beitrag wird für einen Perspektivwechsel plädiert, um mittelfristig persistente Strukturen in den Debatten um das vermeintlich richtige Verhältnis von Markt, Staat und Wettbewerb freizulegen. Erkennbar werden so die Konturen einer , unausgetragenen ' Wirtschaftsordnung, um deren Ausrichtung zwischen den I 9 Ioer Jahren und den späten I940ern erbittert gerungen wurde, und die doch stets in der Schwebe blieb. Die zeitgenössischen Kontroversen lassen sich als Suche nach einem kohärenten Prinzip interpretieren, die erst in der frühen Bundesrepublik ihren einstweiligen Abschluss fand. Denn die zunächst ambitionierten, dann pragmatischen Versuche in der Weimarer Republik, die Beziehungen der privaten Marktakteure zueinander und zum Gemeinwesen auf eine neue Grundlage zu stellen, vermochten im Spannungsfeld von ökonomischen Problemen und tagespolitischen Nöten nur eine fragile, beständig herausgeforderte Struktur auszuformen. Die Lösungsansätze dieses wirtschaftsund verfassungsrechtlichen Ordnungsproblems, das durch die Delegitimierung des klassisch-liberalen wie des zwangswirtschaftlichen Paradigmas infolge des Ersten Weltkrieges aufgeworfen worden war, blieben somit bis in die zweite Nachkriegszeit nur tentativ, ehe sich mit ordoliberalen Ansätzen, durch inhaltliche Häutungen, personale Kontinuitäten und historische Kontingenzen bedingt, ein neues Prinzip durchzusetzen vermochte - und schon bald verklärt wurde.

Dr. Franz Hederer, Johann Wolfgang Goethe Universität, Institut für Rechtsgeschichte, Theodor-W.Adorno-Platz 4, 60629 Frankfurt am Main

Prof. Dr. Kim Christian Priemel, University of Oslo, Department of Archaeology, Conservation and History, Postboks I008, Blindern, 03 I5 Oslo 